\title{
An Asymptotic Expression for the Splitting of Separatrices of the Rapidly Forced Pendulum
}

\author{
Amadeu Delshams ${ }^{1}$ and Teresa M. Seara ${ }^{2}$ \\ ${ }^{1}$ Departament de Matemàtica Aplicada i Anàlisi, Universitat de Barcelona, Gran Via 585, \\ E-08071 Barcelona, Spain \\ 2 Departament de Matemàtica Aplicada I, E.T.S.E.I.B., U. Politècnica de Catalunya, Diagonal \\ 647, E-08028 Barcelona, Spain
}

Received September 1, 1991; in revised form March 16, 1992

Abstract. The measure of the splitting of the separatrices of the rapidly forced pendulum

$$
\ddot{x}+\sin x=\mu \sin \frac{t}{\varepsilon},
$$

is considered as a model problem that has been studied by different authors. Here $\varepsilon, \mu$ are small parameters, $\varepsilon>0$, but otherwise independent. The following formula for the angle $\alpha$ between separatrices is established

$$
\alpha=\frac{\pi}{2 \varepsilon} \frac{\mu}{\cosh \frac{\pi}{2 \varepsilon}}\left[1+O\left(\mu, \varepsilon^{2}\right)\right] .
$$

This formula is also valid for the particular case $\mu=\varepsilon^{p}$, with $p>0, \varepsilon>0$, and agrees with the one provided by the first order Poincaré-Melnikov theory that cannot be applied directly, due to the exponentially small dependence of $\alpha$ on the parameter $\varepsilon$.

\section{Introduction}

Let us consider the equation of the rapidly forced pendulum

$$
\ddot{x}+\sin x=\mu \sin \frac{t}{\varepsilon},
$$

where $\varepsilon, \mu$ are small parameters, $0<\varepsilon<1$, but otherwise independent. This equation can be considered as a model of a two-dimensional integrable system perturbed by a very rapidly oscillatory forcing. Also, performing the change of time $\tau=t / \varepsilon$, it can be considered as a nearly integrable system with slow dynamics:

$$
x^{\prime \prime}+\varepsilon^{2} \sin x=\mu \varepsilon^{2} \sin \tau \quad\left({ }^{\prime}=\frac{d}{d \tau}\right)
$$


Since the forcing in (1.1) is $2 \pi \varepsilon$-periodic, the dynamical properties of this equation can be better visualized with the help of the associated Poincare map $P$, defined in the usual way: if $z=\left(x_{0}, \dot{x}_{0}\right)$ is a point of the plane, and $x(t)$ is the solution of $(1.1)$ satisfying the initial conditions $x(0)=x_{0}, \dot{x}(0)=\dot{x}_{0}$, then $P(z)$ is defined simply as $(x(2 \pi \varepsilon), \dot{x}(2 \pi \varepsilon))$. Since Eq. (1.1) can be written as a (non-autonomous) hamiltonian system, it turns out that $P$ is an area preserving map on the plane.

If $\mu=0$, the phase portrait of $P$ is very well known. We can consider $x$ defined $\bmod 2 \pi$, and thus the phase space is a cylinder, filled up with integral curves of the simple pendulum, consisting of closed orbits, except for one elliptic fixed point at $(0(\bmod 2 \pi), 0)$, one hyperbolic fixed point at $(-\pi(\bmod 2 \pi), 0)$, and two separatrices associated to the hyperbolic point, given by the two homoclinic orbits of the simple pendulum:

$$
\Gamma_{ \pm}=\left\{\left(x_{0}(t), \pm y_{0}(t)\right)\right\}
$$

with

$$
\begin{aligned}
& x_{0}(t)=2 \arctan (\sinh t), \\
& y_{0}(t)=\dot{x}_{0}(t)=\frac{2}{\cosh t} .
\end{aligned}
$$

The phase space looks more complicated if $\mu \neq 0$, at least near the unperturbed separatrices. If $\mu$ is small enough, there is still a hyperbolic fixed point of $P$ near $(-\pi, 0)$, corresponding to a hyperbolic $2 \pi \varepsilon$-periodic orbit of $(1.1)$, as well as the stable and unstable invariant curves $C^{s}, C^{u}$, that lie near the unperturbed separatrices $\Gamma_{ \pm}$. They intersect in the so-called homoclinic points. Due to the special form of Eq. (1.1), they must intersect on a point $z_{h}$ on the axis $\dot{x}$, as well as along its orbit $\left\{P^{n}\left(z_{h}\right), n \in \mathbb{Z}\right\}$. Let us call $\alpha$ the angle between the invariant curves $C^{u}, C^{s}$.

If $\alpha$ is different from zero, the invariant curves intersect transversally at $z_{h}$ and enclose loops whose area $S$ is an invariant, i.e., it does not depend on the homoclinic point $z_{h}$ that has been chosen. The "width" of these loops is measured by the distance between the invariant curves along orthogonal lines to the unperturbed separatrix. We call $d$ the maximum of such distances in the first loop. These three quantities are very related, but only $S$ is an invariant. All they measure the

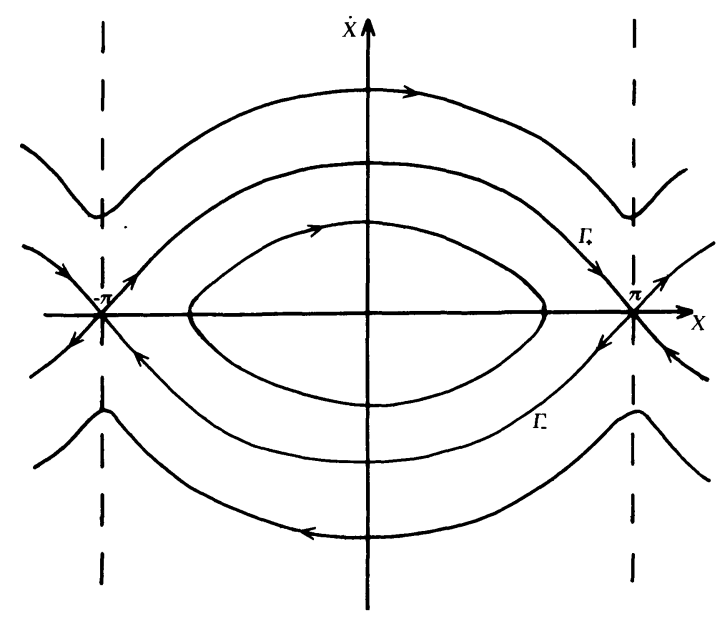

Fig. 1. Phase space of the pendulum equation 


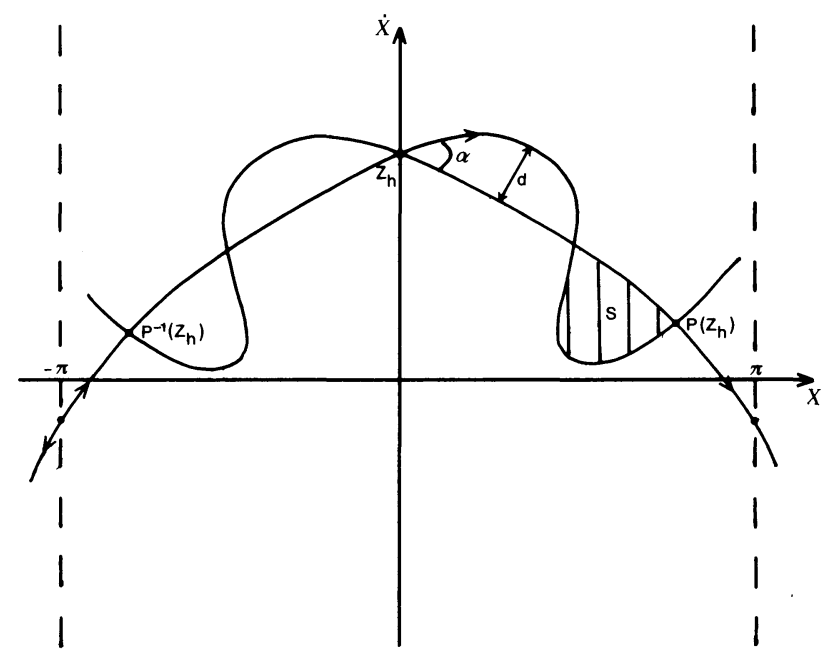

Fig. 2. Phase space of the perturbed pendulum equation

stochastic layer produced by the transversal intersection of $C^{u}, C^{s}$. In fact, this layer is thicker by an algebraic factor in $\varepsilon$, which was derived by B.V. Chirikov and rigorously obtained by V.F. Lazutkin for the standard map in [La91]. The main goal of this paper consists of obtaining asymptotic formulae for such quantities, and is summarized in the following result.

Theorem 1. The Splitting of Separatrices. For $\varepsilon \rightarrow 0, \mu \rightarrow 0$, the following asymptotic formulae hold:

$$
\begin{aligned}
\sin \alpha & =\frac{\pi}{2 \varepsilon} \frac{\mu}{\cosh \frac{\pi}{2 \varepsilon}}\left[1+O(\mu)+O\left(\varepsilon^{2}\right)\right], \\
d & =\frac{\pi \mu}{\cosh \frac{\pi}{2 \varepsilon}}\left[1+O(\mu)+O\left(\varepsilon^{2}\right)\right], \\
S & =\frac{4 \pi \mu \varepsilon}{\cosh \frac{\pi}{2 \varepsilon}}\left[1+O(\mu)+O\left(\varepsilon^{2}\right)\right] .
\end{aligned}
$$

In the above theorem, $\mu, \varepsilon$ are independent parameters. A particular case takes place if one considers $\mu$ as a positive power of $\varepsilon: \mu=\varepsilon^{p}$.

Corollary 2. If $\mu=\varepsilon^{p}$ in Eq. (1.1), with $p>0$, then the following asymptotic formulae hold, where $\gamma=\min \{2, p\}$ :

$$
\begin{aligned}
\sin \alpha & =\frac{\pi}{2} \frac{\varepsilon^{p-1}}{\cosh \frac{\pi}{2 \varepsilon}}\left[1+O\left(\varepsilon^{\gamma}\right)\right], \\
d & =\frac{\pi \varepsilon^{p}}{\cosh \frac{\pi}{2 \varepsilon}}\left[1+O\left(\varepsilon^{\gamma}\right)\right], \\
S & =\frac{4 \pi \varepsilon^{p+1}}{\cosh \frac{\pi}{2 \varepsilon}}\left[1+O\left(\varepsilon^{\gamma}\right)\right] .
\end{aligned}
$$


The main problem in establishing the theorem on the splitting of separatrices comes from the fact that the measured quantities $\sin \alpha, d, S$ are exponentially small in the parameter $\varepsilon$, and because of this, the classical perturbation methods do not apply.

In fact, in a first attempt, one can try to apply directly the so-called PoincaréMelnikov theory, that gives the following formula for the splitting of separatrices

$$
d=\frac{\mu}{2} \max _{s}|M(s)|+O\left(\mu^{2}\right),
$$

where $M(s)=M(s, \varepsilon)$ is the celebrated Poincaré-Melnikov integral

$$
M(s)=\int_{-\infty}^{\infty} \dot{x}_{0}(\sigma+s) \sin \frac{\sigma}{\varepsilon} d \sigma,
$$

that only depends on the unperturbed separatrix and the perturbation, and thus can be calculated using residues, giving rise to the $2 \pi \varepsilon$-periodic in the variable $s$ expression:

$$
M(s)=\frac{2 \pi}{\cosh \frac{\pi}{2 \varepsilon}} \sin \frac{s}{\varepsilon} .
$$

In this way we arrive at the following formula:

$$
d=\frac{\pi \mu}{\cosh \frac{\pi}{2 \varepsilon}}+O\left(\mu^{2}\right),
$$

where the first term gives the correct result, but this first term only can dominate the second term $O\left(\mu^{2}\right)$ if the parameters $\mu, \varepsilon$ are related by

$$
\mu=o\left(\frac{1}{\cosh \frac{\pi}{2 \varepsilon}}\right)=o\left(e^{-\pi / 2 \varepsilon)},\right.
$$

i.e., if $\mu$ is exponentially small with respect to the parameter $\varepsilon$. Thus, this direct approach gives the correct estimate for the splitting of separatrices, but cannot be justified in the case that $\mu, \varepsilon$ are independent small parameters. As we shall see later on, the main point in establishing such result will consist of computing $M(s)$ also for complex values of $s$.

The study of the splitting of separatrices was initiated by H. Poincare in [Po93], and he was aware of the fact that the splitting was not of finite order in the parameter $\varepsilon$ for this kind of singular problems. Later on, Arnold [Ar64] and Melnikov [Me63] developed the so-called Poincaré-Melnikov theory. This theory, as well as the above discussion, can be also found in chapter 4 of the book [GH83] by J. Guckenheimer and P. Holmes. J. Sanders [Sa82] noticed also the problems, previously stated by Poincaré, arising from the direct application of the PoincaréMelnikov integral, when this one depends on the small parameter $\varepsilon$.

Upper bounds for the exponentially small splitting of separatrices in the case of hamiltonian systems with two degrees of freedom were given by A.I. Neishtadt [Ne84], and A. Benseny, A. Delshams [BD87]. Upper bounds for families of area 
preserving diffeomorphisms close to the identity, having homoclinic points, have been obtained by E. Fontich, C. Simó, [FS90], and by E. Fontich [Fo91] for o.d.e.

$$
\ddot{x}=f(x)+\varepsilon^{p} g\left(\frac{t}{\varepsilon}\right),
$$

for $p>-2$, and zero-mean functions $g$. The case of families of volume preserving $q$-dimensional maps, $q>1$, is considered by C. Amick, S.C.E. Ching, L.P. Kadanoff and V. Rom-Kedar [AC89]. V.F. Lazutkin [La84] investigated the standard mapping

$$
\operatorname{SM}(x, y)=\left(x+y^{\prime}, y^{\prime}=y+\varepsilon f(x)\right),
$$

where $f(x)=\sin x$, and was able to give an asymptotic expression for the angle between separatrices

$$
\sin \alpha=\frac{\pi\left|\theta_{1}\right|}{\varepsilon} e^{-\pi^{2} / \sqrt{\varepsilon}}\left[1+O\left(\varepsilon^{1 / 16}\right)\right] ;
$$

where the coefficient $\left|\theta_{1}\right|$ does not depend on $\varepsilon$,

$$
\left|\theta_{1}\right|=1118.82770595 \ldots \text {, }
$$

and was later computed by V.F. Lazutkin, I.G. Schachmannski and M.B. Tabanov [LST89]. For the standard mapping, but with $f(x)$ a polynomial or a trigonometrical polynomial, V.G. Gelfreich, V.F. Lazutkin, and M.B. Tabanov [GLT91] obtained also asymptotic expressions for the splitting of separatrices.

It has to be noticed that the problem for maps is significantly more difficult than for flows, because the linear difference equations that appear in that case do not lead to a computational Poincaré-Melnikov theory, as happens in the case of flows, and the analysis is more intricate (see [La84]).

Concerning our model equation (1.1), it was first considered by P. Holmes, J. Marsden and J. Scheurle [HMS88] in the case $\mu=\delta \varepsilon^{p}$ :

$$
\ddot{x}+\sin x=\delta \varepsilon^{p} \sin \frac{t}{\varepsilon},
$$

and the following lower and upper bounds were derived:

$$
c_{2} \delta \varepsilon^{p} e^{-\pi / 2 \varepsilon} \leqq d \leqq c_{1} \delta \varepsilon^{p} e^{-\pi / 2 \varepsilon},
$$

for $\delta, \varepsilon$ small enough, and $p \geqq 8$. This result was improved by V.G. Gelfreich [Ge90], and he gave the asymptotic expression for the angle only under the assumption $p>5$. Numerical computations performed by C. Simó [Si90], A. Benseny and C. Olivé [BO91] show that the asymptotic expression may be valid for the range of values of the parameter $\mu=\varepsilon^{p}$ for $p \geqq-1$.

The rest of this paper is organized in the following way. In the next two sections, we recall the results about the existence of a $2 \pi \varepsilon$-periodic orbit $\gamma$ of (1.1) near the point $(x, \dot{x})=(-\pi, 0)$. This periodic orbit is hyperbolic and thus the Birkhoff normal form is convergent in a complex neighborhood of $\gamma$, with a radius of convergence not depending on $\varepsilon, \mu$. This analytical normal form provides good parameterizations for the local manifolds associated to $\gamma$, in terms of time $t$ and a complex parameter $s$. The extension theorem of Sect. 4 shows that these parameterizations can be extended, on a strip of values of $t, s$, for instance for the 
unstable manifold, as far as it comes back to the neighborhood of convergence of the normal form. The extension theorem is crucial in our result, and it involves several technicalities as a good choice of the solutions of the variational equation associated to the unperturbed separatrix, as well as a partition of the strip in different regions in order to get this result. Its proof is relegated to Sect. 7. In Sect. 5, the local first integral provided by the analytic normal form is defined, and extended along the invariant manifolds, using the extension theorem. Finally, in Sect. 6, the distance function $\psi(s)$ between invariant manifolds is introduced, following [La84, Ge90], its main properties are proven, and the announced results relating this function with the Poincaré-Melnikov integral follow from the extension theorem and Fourier series arguments.

Our approach for obtaining the exponentially small splitting follows the main ideas of [La84, Ge90], but we do not try to extend the first integral defined in a neighborhood of the periodic orbit. Instead, we use the extension theorem to "come back" to this neighborhood of the periodic orbit, and we use the Poincare-Melnikov integral for complex values of the variable $s$. Our method is applied in this paper only to Eq. (1.1), because it has become "the" model equation for this matter of splitting of separatrices, but we think it can be applied to different kinds of systems with rapidly oscillatory forcing, at least if the singularities of the homoclinic solution on the complex field are uniquely poles.

\section{The Periodic Orbit}

The equation of the rapidly forced pendulum

$$
\ddot{x}+\sin x=\mu \sin \frac{t}{\varepsilon},
$$

can be written as a hamiltonian system:

$$
\begin{aligned}
& \dot{x}=y, \\
& \dot{y}=-\sin x+\mu \sin \frac{t}{\varepsilon},
\end{aligned}
$$

with associated hamiltonian

$$
h\left(x, y, \frac{t}{\varepsilon}, \mu\right)=h^{0}(x, y)-\mu x \sin \frac{t}{\varepsilon},
$$

$h$ being non-autonomous, $2 \pi$-periodic in the variable $\frac{t}{\varepsilon}$, and where

$$
h^{0}(x, y)=\frac{y^{2}}{2}-(\cos x+1)=\frac{y^{2}}{2}-2 \cos ^{2} \frac{x}{2} .
$$

Equation (2.1) is a perturbation, of order $\mu$, of the pendulum equation

$$
\ddot{x}+\sin x=0,
$$

or equivalently, of the hamiltonian system:

$$
\begin{aligned}
& \dot{x}=y, \\
& \dot{y}=-\sin x,
\end{aligned}
$$

associated to the autonomous hamiltonian $h^{0}$. 
Equations (2.2) and (2.6) are $2 \pi$-periodic in the variable $x$, and therefore we can consider this variable defined $\bmod 2 \pi$. System (2.6) has a hyperbolic equilibrium point at $\gamma_{0}=(-\pi, 0)$. The level set $h^{0}=0$ is composed of this hyperbolic point and two homoclinic orbits $\Gamma_{ \pm}=\left\{\left(x_{0}(t), \pm y_{0}(t)\right)\right\}$, given explicitly by:

$$
\begin{aligned}
& x_{0}(t)=2 \arctan (\sinh t), \\
& y_{0}(t)=\frac{2}{\cosh t} .
\end{aligned}
$$

Considering now the full system (2.2), it is well known that it has, for $\mu$ small enough, a hyperbolic $2 \pi \varepsilon$-periodic orbit $\gamma=\left\{\left(x_{p}(t), y_{p}(t)\right)\right\}$ near $\gamma_{0}$.

In fact, rescaling time through $\tau=t / \varepsilon$ and shifting $\gamma_{0}$ to the origin by $\tilde{x}=x+\pi$, we get from (2.1) the equivalent equation

$$
\tilde{x}^{\prime \prime}=\varepsilon^{2} \sin \tilde{x}+\mu \varepsilon^{2} \sin \tau \quad\left({ }^{\prime}=\frac{d}{d \tau}\right),
$$

$2 \pi$-periodic in the time $\tau$. The $2 \pi$-periodic orbit $\gamma$ of $(2.8)$ near the origin can be found using the standard method of successive approximations. Thus, beginning with a $2 \pi$-periodic approximation $\tilde{x}_{0}(\tau)=0$, we can define recursively $2 \pi$-periodic approximations $\tilde{x}_{n}(\tau), \tau \in \mathbb{R}$, by

$$
\begin{aligned}
\tilde{x}_{n+1}(\tau) & =\frac{1}{2 \varepsilon \sinh \pi \varepsilon} \int_{-\pi}^{\pi}(\cosh \varepsilon \sigma-1) f_{n}(\tau+\sigma) d \sigma \\
& =\frac{1}{2 \varepsilon \sinh \pi \varepsilon} \int_{\tau-\pi}^{\tau+\pi}(\cosh [\varepsilon(\tau-\sigma)]-1) f_{n}(\sigma) d \sigma
\end{aligned}
$$

where

$$
f_{n}(\tau)=\varepsilon^{2}\left(\sin \left(\tilde{x}_{n}(\tau)\right)-\tilde{x}_{n}(\tau)\right)+\mu \varepsilon^{2} \sin \tau .
$$

The recurrence (2.9) preserves the symmetry properties of (2.8):

$$
\tilde{x}_{n}(-\tau)=-\tilde{x}_{n}(\tau)=\tilde{x}_{n}(\tau+\pi), \quad n \geqq 0, \tau \in \mathbb{R},
$$

its first non-zero iterate is

$$
\tilde{x}_{1}(\tau)=-\frac{\mu \varepsilon^{2}}{1+\varepsilon^{2}} \sin \tau
$$

and gives, for $\mu$ small enough, the desired uniform convergence of $\left(\tilde{x}_{n}\right)_{n \geqq 0}$ to a $2 \pi$-periodic solution $\tilde{x}_{p}(\tau)=\tilde{x}_{p}(\tau, \mu, \varepsilon)$ of $(2.8)$ satisfying:

$$
\begin{aligned}
& \tilde{x}_{p}(\tau)=\tilde{x}_{1}(\tau)+O\left(\mu^{3} \varepsilon^{8}\right), \\
& \tilde{x}_{p}^{\prime}(\tau)=\tilde{x}_{1}^{\prime}(\tau)+O\left(\mu^{3} \varepsilon^{8}\right) .
\end{aligned}
$$

Remark. From the expression (2.12), one can see that the condition of smallness on $\mu$ is not strictly necessary for the existence of the periodic orbit $\gamma$. For the relevant case, i.e., the existence of a hyperbolic periodic orbit $\gamma$, it is only required that $\mu \varepsilon^{2}$ be small, and therefore $\mu$ can be big (for instance, $\mu=1 / \varepsilon$ ). For more details see [Fo91].

Concerning the original system (2.2), from (2.12) we get the form of the $2 \pi \varepsilon$-periodic orbit $\gamma$, and we summarize all these previous results in the following proposition. 
Proposition 3. Existence of the Periodic Orbit. If $\mu \varepsilon^{2}$ is small enough, system (2.2) has a $2 \pi \varepsilon$-periodic orbit $\gamma=\left\{\left(x_{p}(t), y_{p}(t)\right)\right\}$ near the point $\gamma_{0}=(-\pi(\bmod 2 \pi), 0)$ :

$$
\begin{aligned}
& x_{p}(t)=\tilde{x}_{p}\left(\frac{t}{\varepsilon}\right)-\pi=-\pi-\frac{\mu \varepsilon^{2}}{1+\varepsilon^{2}} \sin \frac{t}{\varepsilon}+O\left(\mu^{3} \varepsilon^{8}\right), \\
& y_{p}(t)=\dot{x}_{p}(t)=\frac{1}{\varepsilon} \tilde{x}_{p}^{\prime}\left(\frac{t}{\varepsilon}\right)=-\frac{\mu \varepsilon}{1+\varepsilon^{2}} \cos \frac{t}{\varepsilon}+O\left(\mu^{3} \varepsilon^{7}\right) .
\end{aligned}
$$

In order to study the local behavior of the periodic orbit, we perform in system (2.2) the canonical change of variables:

$$
\begin{aligned}
& q=x-x_{p}(t), \\
& p=y-y_{p}(t),
\end{aligned}
$$

obtaining the equivalent hamiltonian system

$$
\begin{aligned}
& q^{\prime}=p, \\
& p^{\prime}=-\sin \left(x_{p}(t)+q\right)+\sin x_{p}(t) .
\end{aligned}
$$

This is again a hamiltonian system, with hamiltonian given by $k$, where

$$
k=k(q, p, t, \mu, \varepsilon)=\frac{p^{2}}{2}-\cos \left(x_{p}(t)+q\right)+\cos x_{p}(t)-q \sin x_{p}(t) .
$$

The hamiltonian $k$ depends on the variables $\mu, \varepsilon$, through the periodic orbit $x_{p}(t)=x_{p}(t, \mu, \varepsilon)$, and can be separated in two parts

where

$$
k=k^{0}(q, p)+k^{1}(q, p, t, \mu, \varepsilon),
$$

$$
k^{0}(q, p)=\frac{p^{2}}{2}+\cos q-1=h^{0}(q-\pi, p)=\frac{1}{2}\left(p^{2}-q^{2}\right)+O_{4}(q),
$$

with $\mathrm{O}_{4}(q)$ denoting an analytic function of $q$, beginning with terms of order greater than or equal to 4 in the variable $q$, and

$$
\begin{aligned}
k^{1}:=k-k^{0} & =-\cos \left(x_{p}(t)+q\right)+\cos x_{p}(t)-q \sin x_{p}(t)+1-\cos q \\
& =\left(1+\cos x_{p}(t)\right) \frac{q^{2}}{2}+O\left(\mu \varepsilon^{2} q^{3}\right),
\end{aligned}
$$

where we have used that $x_{p}(t)=-\pi+O\left(\mu \varepsilon^{2}\right)$ (see (2.13)).

Now, the origin $q=p=0$ is a hyperbolic equilibrium point of the hamiltonian system associated to $k^{0}$, and the separatrices given by the equation $k^{0}=0$ are the invariant stable and unstable curves of this equilibrium point. In order to obtain good parameterizations for the invariant manifolds associated to the periodic orbit $\gamma$, i.e., the origin in system (2.15), we shall deal with the (Birkhoff) normal form of $k$ in a neighborhood of the origin.

\section{The Normal Form}

First of all, we can put the linear part of system (2.2) into its normal form, using standard Floquet theory. This is summarized in the following proposition. 
Proposition 4. Linear Normal Form. There exists a linear, $2 \pi \varepsilon$-periodic in time, canonical change

verifying

$$
\begin{aligned}
& q=a_{11}\left(\frac{t}{\varepsilon}, \mu, \varepsilon\right) Q+a_{12}\left(\frac{t}{\varepsilon}, \mu, \varepsilon\right) P \\
& p=a_{21}\left(\frac{t}{\varepsilon}, \mu, \varepsilon\right) Q+a_{22}\left(\frac{t}{\varepsilon}, \mu, \varepsilon\right) P
\end{aligned}
$$

$$
\begin{aligned}
& a_{11}=\frac{1}{c}+O\left(\mu \varepsilon^{2}\right), \quad a_{12}=-\frac{c}{2}+O\left(\mu \varepsilon^{2}\right), \\
& a_{21}=\frac{1}{c}+O\left(\mu \varepsilon^{2}\right), \quad a_{22}=\frac{c}{2}+O\left(\mu \varepsilon^{2}\right),
\end{aligned}
$$

where $c \neq 0$ is an arbitrary constant. With this change and the translation (2.14), the original system (2.2) is transformed into a hamiltonian system:

$$
\begin{aligned}
& \dot{Q}=\frac{\partial K}{\partial P}\left(Q, P, \frac{t}{\varepsilon}, \mu, \varepsilon\right)=\omega Q+\cdots, \\
& \dot{P}=-\frac{\partial K}{\partial Q}\left(Q, P, \frac{t}{\varepsilon}, \mu, \varepsilon\right)=-\omega P+\cdots,
\end{aligned}
$$

whose hamiltonian $K$ is in normal form up to order 2:

with

$$
\begin{aligned}
K\left(Q, P, \frac{t}{\varepsilon}, \mu, \varepsilon\right) & =K^{0}(Q, P)+K^{1}\left(Q, P, \frac{t}{\varepsilon}, \mu, \varepsilon\right) \\
& =\omega Q P+K_{3}\left(Q, P, \frac{t}{\varepsilon}, \mu, \varepsilon\right),
\end{aligned}
$$

$$
K_{3}=O_{3}(Q, P), \quad K^{1}=O\left(\mu \varepsilon^{2}\right),
$$

and $\omega=\omega(\mu, \varepsilon)=1+O\left(\mu^{2} \varepsilon^{4}\right)$.

The $2 \pi \varepsilon$-periodic orbit $\gamma$ of system (2.2) is now placed at the origin in these coordinates $(Q, P)$, and its characteristic exponents are given by $\pm \omega$.

Moreover, it is rather clear that the unperturbed linear change of variables

$$
\begin{aligned}
& q=\frac{1}{c} Q-\frac{c}{2} P, \\
& p=\frac{1}{c} Q+\frac{c}{2} P,
\end{aligned}
$$

transforms the unperturbed hamiltonian $k^{0}$ in its normal form up to order two, i.e., expressing $k^{0}$ in the new variables $Q, P$, it gets the form:

$$
k^{0}(q, p)=K^{0}(Q, P)=Q P+O_{4}(Q, P) .
$$

The hamiltonian $K$ of (3.4) is now ready to perform on it the nonlinear normal form. In our case, this procedure has two important properties.

On the one hand, since the origin of the hamiltonian system associated to $K$ is hyperbolic, the transformation to normal form is convergent in a neighborhood of the origin (see [Mo56, Br71, Br72]). 
On the other hand, as $K$ depends smoothly (in fact analytically) on the parameters $\mu, \varepsilon$, the same will happen to the normal form (see [AKN88, Br89, FS90, Se91]), and, as a consequence, for $\varepsilon, \mu$ small, the radius of convergence for the transformations to normal form can be bounded by a constant $R$ not depending on $\mu, \varepsilon$. We can now collect these results about the nonlinear normal form near $\gamma$ in the following proposition.

Proposition 5. The Nonlinear Normal Form. There exists positive $\varepsilon_{0}, \mu_{0}, R$ and a canonical change of variables:

$$
\begin{aligned}
& Q=\phi\left(X, Y, \frac{t}{\varepsilon}, \mu, \varepsilon\right)=\phi^{0}(X, Y)+\phi^{1}\left(X, Y, \frac{t}{\varepsilon}, \mu, \varepsilon\right), \\
& P=\psi\left(X, Y, \frac{t}{\varepsilon}, \mu, \varepsilon\right)=\psi^{0}(X, Y)+\psi^{1}\left(X, Y, \frac{t}{\varepsilon}, \mu, \varepsilon\right),
\end{aligned}
$$

analytic for $|X|^{2}+|Y|^{2}<2 R, t \in \mathbb{R},|\mu|<\mu_{0}, 0<\varepsilon<\varepsilon_{0}, 2 \pi \varepsilon$-periodic in $t$, that transforms the hamiltonian system (3.3) into the hamiltonian system generated by a hamiltonian $H$ that is in normal form:

with

$$
\begin{aligned}
H(X, Y, \mu, \varepsilon) & =F(X Y, \mu, \varepsilon)=F^{0}(X Y)+F^{1}(X Y, \mu, \varepsilon) \\
& =\omega X Y+F_{2}(X Y, \mu, \varepsilon),
\end{aligned}
$$

$$
\phi^{1}, \psi^{1}, F^{1}=O\left(\mu \varepsilon^{2}\right), \quad F_{2}=O_{2}(X Y) .
$$

This hamiltonian system is given by the equations:

$$
\dot{X}=F^{\prime}(X Y, \mu, \varepsilon) X, \quad \dot{Y}=-F^{\prime}(X Y, \mu, \varepsilon) Y,
$$

where $F^{\prime}(I, \mu, \varepsilon)$ denotes the derivative of $F$ with respect to its first variable I:

$$
\begin{aligned}
F^{\prime}(I, \mu, \varepsilon) & =\frac{\partial F}{\partial I}(I, \mu, \varepsilon)=\frac{d F^{0}}{d I}(I)+\frac{\partial F^{1}}{\partial I}(I, \mu, \varepsilon) \\
& =\omega+F_{2}^{\prime}(I, \mu, \varepsilon) .
\end{aligned}
$$

Notice also that by means of the unperturbed change $\left(\phi^{0}, \psi^{0}\right)$ :

$$
\begin{aligned}
& Q=\phi^{0}(X, Y)=X+\cdots, \\
& P=\psi^{0}(X, Y)=Y+\cdots,
\end{aligned}
$$

the hamiltonian system of unperturbed hamiltonian $K^{0}$ is transformed in the hamiltonian system of hamiltonian $F^{0}$.

System (3.10) can be easily integrated, using the fact that $I=X Y$ is a first integral:

$$
\begin{aligned}
X(t) & =e^{F^{\prime}\left(X_{0} Y_{0}, \mu, \varepsilon\right) t} X_{0}, \\
Y(t) & =e^{-F^{\prime}\left(X_{0} Y_{0}, \mu, \varepsilon\right) t} Y_{0},
\end{aligned}
$$

the solutions being defined for $t \in \mathbb{R}$, and $\left(X_{0}, Y_{0}\right)$ in the complex disk $\left|X_{0}\right|^{2}+\left|Y_{0}\right|^{2}<2 R$. Also note that $F$ is a real analytic function at least for $|I|<R,|\mu|<\mu_{0}$, and $0<\varepsilon<\varepsilon_{0}$. 


\section{Parameterization of the Invariant Manifolds}

For $Y_{0}=0\left(X_{0}=0\right)$ we have the unstable (stable) invariant curve of the origin of (3.10), and the solution along it can be parameterized as

$$
\begin{gathered}
\left(X^{u}(t, s), Y^{u}(t, s)\right)=\left(e^{\omega(t+s)}, 0\right)(\text { unstable curve) }, \\
\left(X^{s}(t, s), Y^{s}(t, s)\right)=\left(0, e^{-\omega(t+s)}\right)(\text { stable curve }),
\end{gathered}
$$

the parameterizations being defined for $t \in \mathbb{R}, s \in \mathbb{C}$.

Returning to the original coordinates $(x, y)$ through the changes (3.7), (3.1) and (2.14), we get the following parameterization for the unstable manifold $W_{\text {loc }}^{u}(\gamma)$ associated to the hyperbolic periodic orbit $\gamma$ :

$$
\begin{aligned}
& x^{u}(t, s)=x_{p}(t)+a_{11}\left(\frac{t}{\varepsilon}\right) Q^{u}(t, s)+a_{12}\left(\frac{t}{\varepsilon}\right) P^{u}(t, s), \\
& y^{u}(t, s)=\dot{x}^{u}(t, s)=y_{p}(t)+a_{21}\left(\frac{t}{\varepsilon}\right) Q^{u}(t, s)+a_{22}\left(\frac{t}{\varepsilon}\right) P^{u}(t, s),
\end{aligned}
$$

where $a_{i j}\left(\frac{t}{\varepsilon}\right)=a_{i j}\left(\frac{t}{\varepsilon}, \mu, \varepsilon\right)$, and $Q^{u}(t, s)=Q^{u}(t, s, \mu, \varepsilon), P^{u}(t, s)=P^{u}(t, s, \mu, \varepsilon)$ are in fact the parameterizations of the unstable manifold associated to the origin of system (3.3). These functions are given by:

$$
\begin{aligned}
& Q^{u}(t, s)=\phi\left(e^{\omega(t+s)}, 0, \frac{t}{\varepsilon}, \mu, \varepsilon\right), \\
& P^{u}(t, s)=\psi\left(e^{\omega(t+s)}, 0, \frac{t}{\varepsilon}, \mu, \varepsilon\right) .
\end{aligned}
$$

The parameterization (4.2) is well defined and analytic for $t \in \mathbb{R}, s \in \mathbb{C}$ with real part of their sum small enough:

$$
\omega(t+\mathfrak{R} s)<-\frac{1}{2} \ln \frac{1}{2 R}:=-\frac{1}{2} T,
$$

and satisfies for these values of $t, s$ the property:

$$
x^{u}(t+2 \pi \varepsilon, s)=x^{u}(t, s+2 \pi \varepsilon),
$$

as a consequence of the $2 \pi$-periodicity of $\phi, \psi$ with respect to the variable $\frac{t}{\varepsilon}$.

Concerning the stable manifold $W_{\text {loc }}^{s}(\gamma)$, we can now use the symmetry properties of system (2.2), and more precisely the fact that the hamiltonian $h$ satisfies

$$
h\left(-x, y,-\frac{t}{\varepsilon}, \mu\right)=h\left(x, y, \frac{t}{\varepsilon}, \mu\right),
$$

i.e., is reversible under the action of the involution

$$
\mathscr{R}:\left(x, y, \frac{t}{\varepsilon}\right) \rightarrow\left(-x, y,-\frac{t}{\varepsilon}\right) \text {. }
$$


Therefore, if $x(t)$ is a solution of Eq. (2.1), $-x(-t)$ is also a solution, and thus $W^{s}(\gamma)=\mathscr{R} W^{u}(\gamma)(\bmod (2 \pi, 0))$, and we can parameterize $W^{s}(\gamma)$ by:

$$
\begin{aligned}
& x^{s}(t, s)=2 \pi-x^{u}(-t,-s), \\
& y^{s}(t, s)=\dot{x}^{s}(t, s)=\dot{x}^{u}(-t,-s)=y^{u}(-t,-s),
\end{aligned}
$$

for

$$
t \in \mathbb{R}, \quad s \in \mathbb{C} \text { such that } \omega(t+\Re s)>\frac{1}{2} \ln \frac{1}{2 R}:=\frac{1}{2} T .
$$

For $\mu=0$, on the one hand parameterization (4.2), (4.3) for $W^{u}\left(\gamma_{0}\right)=\left(h^{0}\right)^{-1}(0)$ becomes

$$
\begin{aligned}
x_{0}^{u}(t, s) & =-\pi+\frac{1}{c} \phi^{0}\left(e^{(t+s)}, 0\right)-\frac{c}{2} \psi^{0}\left(e^{(t+s)}, 0\right) \\
& =-\pi+\frac{1}{c} e^{(t+s)}+O\left(e^{2(t+s)}\right)
\end{aligned}
$$

where we have used (2.13), (3.7), (3.2) and (3.12), but on the other hand we already have the previous parameterization (2.7):

$$
x_{0}(t+s)=2 \arctan (\sinh (t+s))=-\pi+4 e^{(t+s)}+O\left(e^{3(t+s)}\right) .
$$

These two parameterizations coincide if we choose $c=\frac{1}{4}$, and in this way

$$
x_{0}(t+s)=-\pi+4 \phi^{0}\left(e^{(t+s)}, 0\right)-\frac{1}{8} \psi^{0}\left(e^{(t+s)}, 0\right)
$$

With this choice of $c$, from (4.2), (3.2) and (3.9), we obtain

$$
\begin{aligned}
x^{u}(t, s) & =x_{p}(t)+4 \phi^{0}\left(e^{\omega(t+s)}, 0\right)-\frac{1}{8} \psi^{0}\left(e^{\omega(t+s)}, 0\right)+O\left(\mu \varepsilon^{2} e^{\omega(t+s)}\right) \\
& =x_{p}(t)+x_{0}(\omega(t+s))+\pi+O\left(\mu \varepsilon^{2} e^{\omega(t+s)}\right)
\end{aligned}
$$

for $t \in \mathbb{R}, s \in \mathbb{C}$ satisfying (4.4).

Since $\omega=1+O\left(\mu^{2} \varepsilon^{4}\right)$, we can assume, restricting $\mu_{0}$ if necessary, that $|\omega-1|<\frac{1}{2}$. Then

$$
\left|e^{t+s}-e^{\omega(t+s)}\right|<c^{\prime}|\omega-1| e^{(t+\Re s) / 2}
$$

for $t+\mathfrak{R}<\frac{T}{2}$, with $c^{\prime}$ depending only on $T$. From (4.10) we now obtain the following expression for the parameterization of $W_{\text {loc }}^{u}(\gamma)$ near $\gamma$ :

$$
x^{u}(t, s)=x_{p}(t)+x_{0}(t+s)+\pi+O\left(\mu \varepsilon^{2} e^{\omega(t+s)}\right)+O\left(\mu^{2} \varepsilon^{4} e^{(t+s) / 2}\right) .
$$

In the same way we arrive at

$$
y^{u}(t, s)=\dot{x}^{u}(t, s)=y_{p}(t)+y_{0}(t+s)+O\left(\mu \varepsilon^{2} e^{\omega(t+s)}\right)+O\left(\mu^{2} \varepsilon^{4} e^{(t+s) / 2}\right)
$$

for the same set of values of $t, s$, and we get analogous expressions for $x^{s}(t, s), y^{s}(t, s)$, for $t \in \mathbb{R}, s \in \mathbb{C}$ satisfying (4.9). 
We now want to extend these two formulae relating the parameterization of $W_{\mathrm{loc}}^{u}(\gamma)$ to the unperturbed separatrix for other values of $t, s$. However, it has to be noticed that $x_{0}(t+s)$ has a singularity in the complex field for $t+s= \pm \frac{\pi}{2} i$. More precisely, $\dot{x}_{0}(t+s)$ has a simple pole at these points with residue $\mp 2 i$ :

$$
\dot{x}_{0}(t+s)=\frac{\mp 2 i}{t+s \mp \frac{\pi}{2} i}+O\left(t+s \mp \frac{\pi}{2} i\right) .
$$

That means that we will not be able to control the growth of the parameterization for $\mathfrak{I} s= \pm \frac{\pi}{2}$. We will restrict ourselves, following [HMS88], to a complex strip of imaginary width equal to $\frac{\pi}{2}-\varepsilon$. More precisely, for

$$
t \in \mathbb{R}, s \in \mathbb{C}, \quad|\mathfrak{I} s| \leqq \frac{\pi}{2}-\varepsilon, \quad|t+\mathfrak{R} s| \leqq T,
$$

the following extension theorem will ensure us that the parameterization of $W^{u}(\gamma)$ is still defined and $O(\mu)$-near the unperturbed separatrix. We state it first, for the sake of generality, for arbitrary solutions $x(t, s)$ of $(2.1)$.

Theorem 6. Extension Theorem. Let $x_{0}(t+s)=2 \arctan (\sinh (t+s))$ the unperturbed separatrix (2.7), defined for $t \in \mathbb{R},|\mathfrak{I} s|<\frac{\pi}{2}$. Let $s \in \mathbb{C},|\mathfrak{s} s| \leqq \frac{\pi}{2}-\varepsilon$, and $x(t, s)$ a solution of (2.1) defined for $t=t_{0}$, with $t_{0}+\mathfrak{R} s=-T=-\ln \frac{1}{2 R}$, such that:

$$
\begin{aligned}
x\left(t_{0}, s\right)-x_{0}\left(t_{0}+s\right) & =O\left(\mu \varepsilon^{2}\right), \\
\dot{x}\left(t_{0}, s\right)-y_{0}\left(t_{0}+s\right)+\mu \varepsilon \cos \frac{t_{0}}{\varepsilon} & =O\left(\mu \varepsilon^{2}\right) .
\end{aligned}
$$

Then $x(t, s)$ is defined for $-T \leqq t+\Re s \leqq T$, and satisfies:

$$
x(t, s)-x_{0}(t+s)=O(\mu), \quad \dot{x}(t, s)-y_{0}(t+s)=O(\mu) .
$$

By (4.11), (4.12) and the asymptotic expression of $y_{p}(t)$, it is clear that the parameterization $\left(x^{u}(t, s), y^{u}(t, s)\right)$ of $W^{u}(\gamma)$ satisfies the hypothesis of the extension theorem for $t_{0}=-\mathfrak{R} s-T$, and we have:

$$
\begin{aligned}
x^{u}(t, s)-x_{0}(t+s) & =O(\mu), \\
y^{u}(t, s)-y_{0}(t+s)+\mu \varepsilon \cos \frac{t}{\varepsilon} & =O(\mu),
\end{aligned}
$$

for $t, s$ in the strip (4.13).

The extension theorem is a crucial tool in this paper. When $\left(x_{0}(t+s), \dot{x}_{0}(t+s)\right)$ comes back near $\gamma_{0}=(-\pi(\bmod 2 \pi), 0)$ as $t+\mathfrak{R} s$ grows, $\left(x_{0}(t+s), \dot{x}_{0}(t+s)\right)$ reenters the neighborhood of $\gamma$, where the normal form is convergent, and the same 
will happen to the parameterization of $W^{u}(\gamma)$, due to the bounds (4.14) given by the extension theorem. The main point here is that this is valid for a complex strip of $s$ satisfying $|\mathfrak{J} s| \leqq \frac{\pi}{2}-\varepsilon$, and Fourier series methods will be useful, as we shall see later on. The proof of this theorem involves several technicalities, as a good choice of the solutions of the variational equations associated to the separatrix, and also the partition of the strip in different regions, and is deferred to the last section.

Remark. It has to be noticed here that when $s \in \mathbb{R}$ this extension theorem becomes a well known result and the bounds (4.14) are of the same order as the ones at the initial conditions, i.e.,

$$
\begin{aligned}
& x(t, s)-x_{0}(t+s)=O\left(\mu \varepsilon^{2}\right), \\
& \dot{x}(t, s)-y_{0}(t+s)=O\left(\mu \varepsilon^{2}\right)-\mu \varepsilon \cos \frac{t}{\varepsilon},
\end{aligned}
$$

for $-T \leqq t+s \leqq T$, and $t, s \in \mathbb{R}$. (In fact the proof of these bounds is contained in Proposition 7.2 if we take there $s \in \mathbb{R}$.)

\section{The First Integral}

The hamiltonian $H(X, Y, \mu, \varepsilon)=F(X Y, \mu, \varepsilon)$ given by (3.8) is a first integral of system (3.10), analytic at least for complex $X, Y$ satisfying

$$
|X|^{2}+|Y|^{2}<2 R, \quad|\mu|<\mu_{0}, \quad 0<\varepsilon<\varepsilon_{0} .
$$

We recall that $R$ does not depend on $\mu, \varepsilon$. If we denote now

$$
\begin{aligned}
& X=\Phi\left(Q, P, \frac{t}{\varepsilon}, \mu, \varepsilon\right)=\Phi^{0}(Q, P)+\Phi^{1}\left(Q, P, \frac{t}{\varepsilon}, \mu, \varepsilon\right), \\
& Y=\Psi\left(Q, P, \frac{t}{\varepsilon}, \mu, \varepsilon\right)=\Psi^{0}(Q, P)+\Psi^{1}\left(Q, P, \frac{t}{\varepsilon}, \mu, \varepsilon\right),
\end{aligned}
$$

the canonical change of variables inverse of (3.7), it turns out that $\Phi, \Psi$ are $2 \pi$-periodic in the variable $\frac{t}{\varepsilon}$, analytic for

$$
|Q|^{2}+|P|^{2}<2 \tilde{R}, \quad|\mu|<\mu_{0}, \quad 0<\varepsilon<\varepsilon_{0},
$$

and $\Phi^{1}, \Psi^{1}=O\left(\mu \varepsilon^{2}\right)$. Here $\tilde{R}$ does not depend on $\mu, \varepsilon$.

The hamiltonian $H$ induces a first integral $e$ in system (2.2), simply performing the inverse changes of variables of (3.7), (3.1) and (2.14), and therefore given by

$$
e\left(x, y, \frac{t}{\varepsilon}, \mu, \varepsilon\right)=F\left(\Phi\left(Q, P, \frac{t}{\varepsilon}, \mu, \varepsilon\right) \cdot \Psi\left(Q, P, \frac{t}{\varepsilon}, \mu, \varepsilon\right), \mu, \varepsilon\right)
$$

where

$$
\begin{aligned}
& Q=a_{22}\left(\frac{t}{\varepsilon}\right) q-a_{12}\left(\frac{t}{\varepsilon}\right) p, \\
& P=-a_{21}\left(\frac{t}{\varepsilon}\right) q+a_{11}\left(\frac{t}{\varepsilon}\right) p,
\end{aligned}
$$


and

$$
q=x-x_{p}(t), \quad p=y-y_{p}(t)
$$

$e$ is $2 \pi$-periodic in the real variable $\frac{t}{\varepsilon}$, and analytic for complex $x, y$ satisfying

$$
\left|x-x_{p}(t)\right|^{2}+\left|y-y_{p}(t)\right|^{2}<2 r
$$

for $t \in \mathbb{R}$, and $|\mu|<\mu_{0}, 0<\varepsilon<\varepsilon_{0}$. Here $r$ does not depend on $\mu, \varepsilon$.

Due to the fact that $H$ is zero for $X=0$ or $Y=0$, it turns out that the first integral $e$ vanishes on the periodic orbit $\gamma$ and on the invariant manifolds $W_{\text {loc }}^{u}(\gamma), W_{\text {loc }}^{s}(\gamma)$. Moreover, taking into account the form (3.8) of $H$, as well as the expressions (5.1), (3.5), and (3.1) for the canonical changes, it follows that

$$
\begin{aligned}
e\left(x, y, \frac{t}{\varepsilon}, \mu, \varepsilon\right)= & F^{0}\left(\Phi^{0}\left(\frac{\pi+x+y}{8}, 4(y-x-\pi)\right)\right. \\
& \left.\times \Psi^{0}\left(\frac{\pi+x+y}{8}, 4(y-x-\pi)\right)\right)+O\left(\mu \varepsilon^{2}\right) \\
= & h^{0}(x, y)+O\left(\mu \varepsilon^{2}\right),
\end{aligned}
$$

on the domain (5.5) of definition of $e$.

\section{The Distance Between Invariant Manifolds}

The first integral $e$ is well defined and vanishes on $W_{\text {loc }}^{s}(\gamma)$ :

$$
e\left(x^{s}(t, s), y^{s}(t, s), \frac{t}{\varepsilon}, \mu, \varepsilon\right)=0
$$

for $\omega(t+\mathfrak{R} s)>\frac{T}{2}, 0<\varepsilon<\varepsilon_{0}$.

Now, by means of the extension theorem, $e$ can also be defined on the parameterization for the unstable manifold for $t+\mathfrak{R}$ big enough, since $x^{u}(t, s)$ is near to the separatrix $x_{0}(t+s)$, and this one comes back at a distance of order of $\mu \varepsilon^{2}$ of $x_{p}(t)$ for $t+\mathfrak{R} s$ big. More precisely, we can assume that $R$ has been chosen in such a way that

$$
\left|x_{0}(t+s)-\pi\right|^{2}+\left|\dot{x}_{0}(t+s)\right|^{2}<r
$$

for $t+\mathfrak{R}>\frac{T}{2}$. Then, restricting $\mu_{0}$ if necessary, we can assume that

$$
\left|x^{u}(t, s)-x_{p}(t)\right|^{2}+\left|y^{u}(t, s)-y_{p}(t)\right|^{2}<2 r
$$

for

$$
t \in \mathbb{R}, \quad \frac{T}{2}<t+\mathfrak{R} s<T, \quad|\mathfrak{I} s| \leqq \frac{\pi}{2}-\varepsilon=: r_{\varepsilon}
$$

and $|\mu|<\mu_{0}, 0<\varepsilon<\varepsilon_{0}$. 
As a consequence, $\left(x^{u}(t, s), y^{u}(t, s)\right)$ lies on the domain of definition (5.5) of $e$ for these values of $t, s$, and therefore $e$ can be extended along the orbits $\left(x^{u}(t, s), y^{u}(t, s)\right)$ for $t+\mathfrak{R} s \leqq \frac{T}{2}$, simply taking constant values along these trajectories.

Now following [La84, Ge90], we are finally led to the function that will measure the distance between the invariant manifolds $W^{u}(\gamma), W^{s}(\gamma)$. Let us define

$$
\begin{aligned}
\psi(t, s, \mu, \varepsilon) & =e\left(x^{u}(t, s), y^{u}(t, s), \frac{t}{\varepsilon}, \mu, \varepsilon\right)-e\left(x^{s}(t, s), y^{s}(t, s), \frac{t}{\varepsilon}, \mu, \varepsilon\right) \\
& =e\left(x^{u}(t, s), y^{u}(t, s), \frac{t}{\varepsilon}, \mu, \varepsilon\right),
\end{aligned}
$$

on the domain (6.1). Since $e$ is a first integral, it is constant along trajectories of system (2.2) and consequently $\psi$ does not depend on $t$. We will write simply

$$
\psi(s)=\psi(s, \mu, \varepsilon)=\psi(t, s, \mu, \varepsilon),
$$

for

$$
s \in \mathbb{C}, \quad|\mathfrak{T} s|<\frac{\pi}{2}-\varepsilon, \quad|\mu|<\mu_{0}, \quad 0<\varepsilon<\varepsilon_{0} .
$$

The function $\psi$ gives a measure of the distance between the invariant manifolds $W^{u}(\gamma), W^{s}(\gamma)$. It was introduced by V.F. Lazutkin in [La84], and used in [Ge90, GLT90, LST89, FS90]. A similar and previous approach takes place in [Zi82]. The next proposition establishes the relation between $\psi$ and the Poincaré-Melnikov integral.

Proposition 7. For $|\mathfrak{J} s| \leqq r_{\varepsilon}=\frac{\pi}{2}-\varepsilon$, we have

$$
\psi(s)=\mu\left[M(s)+O(\mu)+O\left(\varepsilon^{2}\right)\right],
$$

where $M(s)$ is the Poincaré-Melnikov integral:

$$
M(s)=\int_{-\infty}^{\infty} y_{0}(\sigma+s) \sin \frac{\sigma}{\varepsilon} d \sigma=\frac{2 \pi}{\cosh \frac{\pi}{2 \varepsilon}} \sin \frac{s}{\varepsilon} .
$$

Proof. From (5.6) and the definition (6.2) of $\psi$, it follows that

$$
\psi(s)=h^{0}\left(x^{u}(t, s), y^{u}(t, s)\right)-h^{0}\left(x^{s}(t, s), y^{s}(t, s)\right)+O\left(\mu \varepsilon^{2}\right)
$$

for $|\mathfrak{J} s| \leqq r_{\varepsilon}, \frac{T}{2} \leqq t+\mathfrak{R} s \leqq T$.

Introducing now the functions

$$
\Delta^{\beta}(t, s)=h^{0}\left(x^{\beta}(t, s), y^{\beta}(t, s)\right)-h^{0}\left(x_{p}(t), y_{p}(t)\right)
$$

for $\beta=s, u ; t \in \mathbb{R},|\mathfrak{J} s| \leqq r_{\varepsilon}$, and

$$
\Delta(t, s)=\Delta^{u}(t, s)-\Delta^{s}(t, s),
$$


relation (6.6) becomes

$$
\psi(s)=\Delta^{u}(t, s)-\Delta^{s}(t, s)+O\left(\mu \varepsilon^{2}\right)=\Delta(t, s)+O\left(\mu \varepsilon^{2}\right) .
$$

We can differentiate (6.7) with respect to the time $t$, obtaining

$$
\dot{\Delta}^{\beta}(t, s):=\frac{\partial \Delta^{\beta}}{\partial t}(t, s)=\mu\left(y^{\beta}(t, s)-y_{p}(t)\right) \sin \frac{t}{\varepsilon}, \quad \beta=s, u .
$$

By the asymptotic properties (4.11), (4.12) of $x^{\beta}(t, s)$ it turns out that

$$
\Delta^{\beta}(t, s) \rightarrow 0 \text { as } t \rightarrow+\infty,-\infty \quad \text { if } \beta=s, u \text {, respectively, }
$$

and consequently

$$
\Delta(t, s)=\mu \int_{-\infty}^{t}\left(y^{u}(\sigma, s)-y_{p}(\sigma)\right) \sin \frac{\sigma}{\varepsilon} d \sigma+\mu \int_{t}^{\infty}\left(y^{s}(\sigma, s)-y_{p}(\sigma)\right) \sin \frac{\sigma}{\varepsilon} d \sigma .
$$

We can now use the approximations provided by (4.12) and (4.14) to compute the above integrals in terms of $y_{0}(\sigma+s)$, obtaining, for instance for $t=T-\mathfrak{R} s$ :

$$
\Delta(t, s)=\mu M(s)+O\left(\mu^{2}\right) \text {. }
$$

Inserting (6.12) in (6.8), we get (6.4).

We have now all the ingredients needed for the proof of Theorem 1. During the rest of this section, $s$ will denote a real number. Using properties (4.5) and the fact that $\psi$ does not depend on $t$, it follows that $\psi$ is a $2 \pi \varepsilon$-periodic function. $\psi$ is also analytic in the domain (6.3) due to the analyticity of the first integral $e$ and the parameterization of the invariant manifolds.

Let us consider now the Fourier expansion of $\psi$ :

$$
\psi(s)=\sum_{k} \psi_{k} e^{i \frac{k}{\varepsilon} s}
$$

with Fourier coefficients

$$
\begin{aligned}
\psi_{k}=\frac{1}{2 \pi \varepsilon} \int_{0}^{2 \pi \varepsilon} \psi(s) e^{-i \frac{k}{\varepsilon} s} d s & =\frac{e^{k \frac{r_{\varepsilon}}{\varepsilon}}}{2 \pi \varepsilon} \int_{0}^{2 \pi \varepsilon} \psi\left(s+i r_{\varepsilon}\right) e^{-i \frac{k}{\varepsilon} s} d s \\
& =\frac{e^{-k \frac{r_{\varepsilon}}{\varepsilon}}}{2 \pi \varepsilon} \int_{0}^{2 \pi \varepsilon} \psi\left(s-i r_{\varepsilon}\right) e^{-i \frac{k}{\varepsilon} s} d s
\end{aligned}
$$

We compute the first Fourier coefficients $\psi_{1}, \psi_{-1}$ using (6.4), (6.5):

$$
\begin{aligned}
\psi_{-1} & =\frac{e^{-\frac{r_{\varepsilon}}{\varepsilon}}}{2 \pi \varepsilon} \int_{0}^{2 \pi \varepsilon} \psi\left(s+i r_{\varepsilon}\right) e^{i \frac{s}{\varepsilon}} d s \\
& =\frac{\mu e^{-\frac{r_{\varepsilon}}{\varepsilon}}}{2 \pi \varepsilon} \int_{0}^{2 \pi \varepsilon}\left[M\left(s+i r_{\varepsilon}\right)+O(\mu)+O\left(\varepsilon^{2}\right)\right] e^{i \frac{s}{\varepsilon}} d s \\
& =\frac{i \pi \mu}{\cosh \frac{\pi}{2 \varepsilon}}\left[1+O\left(\mu, \varepsilon^{2}\right)\right] ;
\end{aligned}
$$


analogously,

$$
\psi_{1}=\frac{-i \pi \mu}{\cosh \frac{\pi}{2 \varepsilon}}\left[1+O\left(\mu, \varepsilon^{2}\right)\right]
$$

The higher order Fourier coefficients can be simply estimated, using (6.13) if $k<-1$, and (6.14) if $k>1$, by

$$
\begin{aligned}
\psi_{k} & =\frac{1}{2 \pi \varepsilon} \int_{0}^{2 \pi \varepsilon} \psi(s) e^{-i \frac{k}{\varepsilon} s} d s=\frac{e^{-|k| \frac{r_{\varepsilon}}{\varepsilon}}}{2 \pi \varepsilon} \int_{0}^{2 \pi \varepsilon} \psi\left(s \pm i r_{\varepsilon}\right) e^{-i \frac{k}{\varepsilon} s} d s \\
& =\frac{\mu e^{-|k| \frac{r_{\varepsilon}}{\varepsilon}}}{2 \pi \varepsilon} \int_{0}^{2 \pi \varepsilon}\left[M\left(s \pm i r_{\varepsilon}\right)+O(\mu)+O\left(\varepsilon^{2}\right)\right] e^{i \frac{k}{\varepsilon} s} d s \\
& =O\left(\mu e^{-|k| \frac{\pi}{2 \varepsilon}}\right) \text { for }|k|>1,
\end{aligned}
$$

and in this way we arrive at the formulae:

$$
\begin{aligned}
& \psi(s)=\psi_{0}+\sum_{k \neq 0} \psi_{k} e^{i \frac{k}{\varepsilon} s}=\psi_{0}+\frac{2 \pi \mu}{\cosh \frac{\pi}{2 \varepsilon}}\left[\sin \frac{s}{\varepsilon}+O(\mu)+O\left(\varepsilon^{2}\right)\right], \\
& \psi^{\prime}(s)=\sum_{k \neq 0} i \frac{k}{\varepsilon} \psi_{k} e^{i \frac{k}{\varepsilon} s}=\frac{2 \pi \mu}{\varepsilon \cosh \frac{\pi}{2 \varepsilon}}\left[\cos \frac{s}{\varepsilon}+O(\mu)+O\left(\varepsilon^{2}\right)\right],
\end{aligned}
$$

for $s \in \mathbb{R}$.

In order to prove Theorem 1 we only need to compute the coefficient $\psi_{0}$, and to relate the function $\psi$ with the quantities $d, \sin \alpha, S$. Inserting (6.18) in the expressions c), d), e) given by the next proposition we obtain the asymptotic formulae for these quantities and Theorem 1 is completely proven.

Proposition 8. The function $\psi$ satisfies the following properties:

a) $\psi\left(s_{0}\right)=0$ for some real $s_{0}=O\left(\mu \varepsilon^{2}\right)$, and therefore we can consider $\psi(0)=0$ performing a translation in the parameter $s$.

b) If $\vec{x}^{u}\left(t, s_{1}\right)=\vec{x}^{s}\left(t, s_{2}\right)$ (giving an homoclinic connection), then $\psi\left(s_{1}\right)=0$, and

$$
\psi^{\prime}\left(s_{1}\right)=\frac{\partial \vec{x}^{s}}{\partial s}\left(t, s_{2}\right) \wedge \frac{\partial \vec{x}^{u}}{\partial s}\left(t, s_{1}\right)=\left\|\frac{\partial \vec{x}^{u}}{\partial s}\left(t, s_{1}\right)\right\|\left\|\frac{\partial \vec{x}^{s}}{\partial s}\left(t, s_{2}\right)\right\| \sin \Theta\left(t, s_{1}, s_{2}\right),
$$

where $\vec{x}^{\beta}(t, s):=\left(x^{\beta}(t, s), y^{\beta}(t, s)\right), \beta=s, u, \wedge$ denotes the exterior product on $\mathbb{R}^{2}$, and $\Theta\left(t, s_{1}, s_{2}\right)$ is the angle between $\vec{x}^{u}\left(t, s_{1}\right)$ and $x^{s}\left(t, s_{2}\right)$.

c) $\psi^{\prime}(0)=\left[4+O\left(\mu \varepsilon^{2}\right)\right] \sin \alpha$.

d) $S=\int_{0}^{\pi \varepsilon} \psi(s) d s, \psi_{0}=\int_{0}^{2 \pi \varepsilon} \psi(s) d s=0$.

e) $d=\frac{1}{2} \max |\psi(s)|\left[1+O\left(\mu \varepsilon^{2}\right)\right]$.

Proof. Let $s_{0} \in \mathbb{R}$ be the value of $s$ such that $\left(x^{u}\left(0, s_{0}\right), y^{u}\left(0, s_{0}\right)\right)$ belongs to the line of fixed points of the involution (4.7), i.e.:

$$
x^{u}\left(0, s_{0}\right)=0
$$


then $\left(x^{u}\left(t, s_{0}\right), y^{u}\left(t, s_{0}\right)\right)$ is the homoclinic orbit of Eq. (1.1), because the symmetry (4.8) implies that

$$
x^{u}\left(t, s_{0}\right)=x^{s}\left(t,-s_{0}\right), \quad y^{u}\left(t, s_{0}\right)=y^{s}\left(t,-s_{0}\right), \quad t \in \mathbb{R} .
$$

In order to know the value $s_{0}$ we have to solve Eq. (6.19). Since $s \in \mathbb{R}$ we can use property (4.15) for $t+s \leqq T$, and from the variational equations satisfied by the derivatives $\left(\frac{\partial x^{u}}{\partial s}(t, s), \frac{\partial y^{u}}{\partial s}(t, s)\right)$ :

$$
\begin{aligned}
& \frac{d}{d t}\left(\frac{\partial x^{u}}{\partial s}\right)=\frac{\partial y^{u}}{\partial s} \\
& \frac{d}{d t}\left(\frac{\partial y^{u}}{\partial s}\right)=-\cos \left(x^{u}(t, s)\right) \cdot \frac{\partial x^{u}}{\partial s}=-\cos \left(x_{0}(t+s)\right) \cdot \frac{\partial x^{u}}{\partial s}+O\left(\mu \varepsilon^{2}\right) \cdot \frac{\partial x^{u}}{\partial s}
\end{aligned}
$$

it follows

$$
\begin{aligned}
& \frac{\partial x^{u}}{\partial s}=\frac{\partial x_{0}}{\partial s}(t+s)+O\left(\mu \varepsilon^{2}\right), \\
& \frac{\partial y^{u}}{\partial s}=\frac{\partial y_{0}}{\partial s}(t+s)+O\left(\mu \varepsilon^{2}\right)
\end{aligned}
$$

for $t+s \leqq T$ and therefore

$$
s_{0}=0+O\left(\mu \varepsilon^{2}\right)
$$

Now, we can change the parameterization of the invariant manifolds performing the translation $s-s_{0}$ on the unstable manifold and consequently, by (4.8), $s+s_{0}$ on the stable one. Due to (6.21), the asymptotic properties (4.11), (4.12) do not change under this translation. From now on, we will consider that we have chosen these parameterizations, and then we have that

$$
\begin{aligned}
& x^{u}(t, 0)=x^{s}(t, 0), \\
& y^{u}(t, 0)=y^{s}(t, 0),
\end{aligned}
$$

and thus $\psi(0)=0$.

b) We differentiate (6.2) at the point $s_{1}$ :

$$
\psi^{\prime}\left(s_{1}\right)=\frac{\partial e}{\partial x}\left(x^{u}\left(t, s_{1}\right), y^{u}\left(t, s_{1}\right), \frac{t}{\varepsilon}\right) \frac{\partial x^{u}}{\partial s}\left(t, s_{1}\right)+\frac{\partial e}{\partial y}\left(x^{u}\left(t, s_{1}\right), y^{u}\left(t, s_{1}\right), \frac{t}{\varepsilon}\right) \frac{\partial y^{u}}{\partial s}\left(t, s_{1}\right) .
$$

Using the normal form theorem given in Propositions 4 and 5, the change of variables

$$
(x, y) \mapsto(s, e)
$$

is symplectic, and one can check that

$$
\begin{aligned}
& e\left(x^{s}(t, s), y^{s}(t, s), t\right)=0, \\
& s\left(x^{s}(t, s), y^{s}(t, s), t\right)=s .
\end{aligned}
$$


Differentiating these two equations and using that the change is area preserving we obtain:

$$
\begin{aligned}
& \frac{\partial x^{s}}{\partial s}\left(t, s_{2}\right)=\frac{\partial e}{\partial y}\left(x^{s}\left(t, s_{2}\right), y^{s}\left(t, s_{2}\right), \frac{t}{\varepsilon}\right)=\frac{\partial e}{\partial y}\left(x^{u}\left(t, s_{1}\right), y^{u}\left(t, s_{1}\right), \frac{t}{\varepsilon}\right) \\
& \frac{\partial y^{s}}{\partial s}\left(t, s_{2}\right)=-\frac{\partial e}{\partial x}\left(x^{s}\left(t, s_{2}\right), y^{s}\left(t, s_{2}\right), \frac{t}{\varepsilon}\right)=-\frac{\partial e}{\partial x}\left(x^{u}\left(t, s_{1}\right), y^{u}\left(t, s_{1}\right), \frac{t}{\varepsilon}\right)
\end{aligned}
$$

and inserting these equalities in the formula of $\psi^{\prime}\left(s_{1}\right)$, we get the required expression:

$$
\psi^{\prime}\left(s_{1}\right)=-\frac{\partial y^{s}}{\partial s}\left(t, s_{2}\right) \frac{\partial x^{u}}{\partial s}\left(t, s_{1}\right)+\frac{\partial x^{s}}{\partial s}\left(t, s_{2}\right) \frac{\partial y^{u}}{\partial s}\left(t, s_{1}\right)
$$

c) is a direct consequence of a), b), and formula (6.20).

d) Consider the Poincare map $P$ defined in the introduction. Using property (4.5), it follows

$$
\begin{aligned}
P\left(x^{s}(0, s), y^{s}(0, s)\right) & =\left(x^{s}(2 \pi \varepsilon, s), y^{s}(2 \pi \varepsilon, s)\right) \\
& =\left(x^{s}(0, s+2 \pi \varepsilon), y^{s}(0, s+2 \pi \varepsilon)\right),
\end{aligned}
$$

and a similar property for the unstable manifold. This means that the invariant stable and unstable curves $C^{\beta}, \beta=s, u$ for the Poincare map are given by

$$
C^{\beta}=\left\{\left(x^{\beta}(0, s), y^{\beta}(0, s)\right)\right\}, \quad s \in \mathbb{R} .
$$

The points $z_{h}=\left(x^{s}(0,0), y^{s}(0,0)\right)$ and $P\left(z_{h}\right)=\left(x^{s}(0,2 \pi \varepsilon), y^{s}(0,2 \pi \varepsilon)\right)$ are homoclinic points of $P$, and due to the fact that $P$ preserves the orientation we have another homoclinic point between them. Consequently the function $\psi$ has one zero in ] $0,2 \pi \varepsilon$ [. Due to the symmetries (4.8) and the periodicity of $\psi$ this new point is given by $\left(x^{s}(0, \pi \varepsilon), y^{s}(0, \pi \varepsilon)\right)$, and therefore $\psi(\pi \varepsilon)=0$.

Since $P$ preserves area, the area of the loop (see Fig. 2) is given by

$$
S=\int_{x^{s}(0, \pi \varepsilon)}^{x^{s}(0,2 \pi \varepsilon)}\left(y^{s}(x)-y^{u}(x)\right) d x=\int_{x^{s}(0,-\pi \varepsilon)}^{x^{s}(0,0)}\left(y^{s}(x)-y^{u}(x)\right) d x,
$$

where $y=y^{\beta}(x)$ is the explicit expression of the curve $C^{\beta}$ in terms of the variable $x, \beta=s, u$. By the symmetry we can also write

$$
S=\int_{x^{s}(0,0)}^{x^{s}(0, \pi \varepsilon)}\left(y^{u}(x)-y^{s}(x)\right) d x=\iint_{D} d x d y
$$

where $D$ is the set $D=\left\{(x, y) \mid x^{s}(0,0) \leqq x \leqq x^{s}(0, \pi \varepsilon), y^{s}(x) \leqq y \leqq y^{u}(x)\right\}$. Performing the area preserving change of variables (6.23) and introducing

$$
D^{\prime}=\left\{(s, e) \mid 0 \leqq s \leqq \pi \varepsilon, e\left(x^{s}(0, s), y^{s}(0, s), 0\right) \leqq e \leqq e\left(x^{u}(0, s), y^{u}(0, s), 0\right)\right\},
$$

the area $S$ is given by

$$
S=\iint_{D^{\prime}} d s d e=\int_{0}^{\pi \varepsilon} \psi(s) d s
$$


Using again the symmetry, one easily gets

$$
\psi_{0}=\int_{0}^{2 \pi \varepsilon} \psi(s) d s=0 .
$$

e) follows from the mean value theorem applied to $\psi$.

\section{Proof of the Extension Theorem}

The proof of Theorem 6 will be performed through two propositions that will assure the extension of $x(t, s)$, in principle only defined for $t+\mathfrak{R} s=-T$. The first proposition gives the whole extension of $x(t, s)$ for $t+\mathfrak{R} s \leqq T$, but only if $0 \leqq \mathfrak{J} s \leqq \frac{\pi}{2}-\varepsilon^{1 / 2}$. For the values of $s$ with $\frac{\pi}{2}-\varepsilon^{1 / 2} \leqq \mathfrak{J} s \leqq \frac{\pi}{2}-\varepsilon$ this proposition only assures the extension of $x(t, s)$ up to a distance of order $\varepsilon^{1 / 2}$ to the singularity of $x_{0}(t+s)$, or equivalently for $t+\mathfrak{R} s \leqq \varepsilon^{1 / 2}$. With the second one we will be able to enlarge the domain for these values of $\mathfrak{I}$ s up to $t+\mathfrak{R} s \leqq T$. These two propositions are also true for $\varepsilon-\frac{\pi}{2} \leqq \mathfrak{I} s \leqq 0$, and putting this altogether, Theorem 6 will be proven.

In order to carry out this procedure we will compare the solution $x(t, s)$ with the homoclinic solution $x_{0}(t+s)$ of the unperturbed equation (2.5), and thus we introduce:

$$
\left\{\begin{array}{l}
\xi(t, s):=x(t, s)-x_{0}(t+s), \\
\eta(t, s):=\dot{x}(t, s)-y_{0}(t+s) .
\end{array}\right.
$$

The system of differential equations verified by $(\xi(t, s), \eta(t, s))$ with respect to the variable $t$ is:

$$
\begin{aligned}
& \dot{\xi}=\eta \\
& \dot{\eta}=-\sin \left(\xi+x_{0}(t+s)\right)+\mu \sin \frac{t}{\varepsilon}+\sin x_{0}(t+s) .
\end{aligned}
$$

In order to study this system it is very convenient to write it as:

$$
\begin{aligned}
& \dot{\xi}=\eta, \\
& \dot{\eta}=-\cos x_{0}(t+s) \cdot \xi+\mu \sin \frac{t}{\varepsilon}+g(t+s, \xi),
\end{aligned}
$$

where

$$
g(\tau, \xi):=-\sin \left(\xi+x_{0}(\tau)\right)+\sin x_{0}(\tau)+\cos x_{0}(\tau) \cdot \xi .
$$

The initial conditions for a value $t_{0}=-T-\Re s$ are

$$
\begin{aligned}
\xi\left(t_{0}, s\right) & =x\left(t_{0}, s\right)-x_{0}\left(t_{0}+s\right) \\
\eta\left(t_{0}, s\right) & =\dot{x}\left(t_{0}, s\right)-y_{0}\left(t_{0}+s\right) \\
& =-\mu \varepsilon \cos \frac{t_{0}}{\varepsilon}+\dot{x}\left(t_{0}, s\right)-y_{0}\left(t_{0}+s\right)+\mu \varepsilon \cos \frac{t_{0}}{\varepsilon}
\end{aligned}
$$


and taking into account the hypothesis of Theorem 6 the following bounds hold:

$$
\begin{aligned}
& \left|\xi\left(t_{0}, s\right)+\mu \varepsilon^{2} \sin \frac{t_{0}}{\varepsilon}\right| \leqq c_{0} \mu \varepsilon^{2}, \\
& \left|\eta\left(t_{0}, s\right)+\mu \varepsilon \cos \frac{t_{0}}{\varepsilon}\right| \leqq c_{0} \mu \varepsilon^{2},
\end{aligned}
$$

$c_{0}$ being a constant independent of $\mu, \varepsilon$.

We will look for the solution of system (7.2) with initial conditions (7.4). First of all we seek for a fundamental matrix of the corresponding homogeneous linear system. This is a crucial point, since an adequate fundamental matrix will give the best bounds for $(\xi(t), \eta(t))$.

The linear system:

$$
\begin{aligned}
& \dot{\xi}=\eta, \\
& \dot{\eta}=-\cos x_{0}(t+s) \cdot \xi,
\end{aligned}
$$

can be integrated using the fact that $\left(y_{0}(t+s), \dot{y}_{0}(t+s)\right)$ is a solution of (7.6). Another independent solution can be obtained in the form: $\xi=y_{0} \cdot W, \eta=\dot{\xi}$ with:

$$
W(\tau)=\int_{a}^{\tau} \frac{1}{y_{0}^{2}(\sigma)} d \sigma
$$

It is very important to choose adequately the parameter " $a$." We will consider first the case $0 \leqq \mathfrak{I} s \leqq \frac{\pi}{2}$. We note that $y_{0}(t+s)$ has a simple pole at the point $t+s=\frac{\pi}{2} i$

$$
y_{0}(t+s)=\dot{x}_{0}(t+s)=\frac{2}{\cosh (t+s)}=\frac{-2 i}{t+s-\frac{\pi}{2} i}+O\left(t+s-\frac{\pi}{2} i\right) \text {. }
$$

As we want a good second solution of the linear system (7.6) near $\tau:=t+s=\frac{\pi}{2} i$ we choose $a=\frac{\pi}{2} i$. In this way $W(\tau)$ has a triple zero at $\tau=\frac{\pi}{2} i$, and $y_{0}(\tau) W(\tau)$ has a double zero at $\tau=\frac{\pi}{2} i$.

Introducing:

$$
\begin{aligned}
& \Psi(\tau):=\frac{1}{2} y_{0}(\tau)=\operatorname{sech}(\tau), \\
& \Phi(\tau):=\Psi(\tau) W(\tau)=\frac{1}{2}\left(\left(\tau-\frac{\pi}{2} i\right) \operatorname{sech}(\tau)+\sinh (\tau)\right),
\end{aligned}
$$

the associated fundamental matrix $M(\tau)=M(t+s)$ of $(7.6)$ is:

$$
\left(\begin{array}{ll}
\Psi(\tau) & \Phi(\tau) \\
\Psi^{\prime}(\tau) & \Phi^{\prime}(\tau)
\end{array}\right)
$$


and the fundamental solution $\varphi(t, \sigma)$ of (7.6) satisfying $\varphi(t, t)=I d$ is given by

$$
\varphi(t, \sigma)=M(t+s) M(\sigma+s)^{-1} .
$$

Expanding the functions $\Phi, \Psi$ near $\tau=\frac{\pi}{2} i$ we get:

$$
\begin{aligned}
& \Psi(\tau)=-\frac{i}{\tau-\frac{\pi}{2} i}\left(1+O\left(\tau-\frac{\pi}{2} i\right)^{2}\right) \\
& \Phi(\tau)=\frac{i}{3}\left(\tau-\frac{\pi}{2} i\right)^{2}\left(1+O\left(\left(\tau-\frac{\pi}{2} i\right)^{2}\right)\right) .
\end{aligned}
$$

That means that the fundamental matrix $M(\tau)$ behaves near the pole as:

$$
\left(\begin{array}{cc}
\frac{-i}{\tau-\frac{\pi}{2} i} & \frac{i}{3}\left(\tau-\frac{\pi}{2} i\right)^{2} \\
\frac{i}{\left(\tau-\frac{\pi}{2} i\right)^{2}} & \frac{2 i}{3}\left(\tau-\frac{\pi}{2} i\right)
\end{array}\right)
$$

Returning to system (7.2) we can easily write the solution $(\xi(t, s), \eta(t, s))=z(t, s)$ with initial condition $z\left(t_{0}, s\right)$ as:

$$
z(t, s)=z_{\text {in }}\left(t_{0}, t, s\right)+\int_{t_{0}}^{t} \varphi(t, \sigma) F(\xi(\sigma, s), \sigma, s) d \sigma
$$

with

$$
z_{\text {in }}\left(t_{0}, t, s\right):=\varphi\left(t, t_{0}\right) z\left(t_{0}, s\right)
$$

and

$$
F(\xi, t, s)=\left(\begin{array}{c}
F_{1}(\xi, t, s) \\
F_{2}(\xi, t, s)
\end{array}\right):=\left(\begin{array}{c}
0 \\
\mu \sin \frac{t}{\varepsilon}+g(t+s, \xi)
\end{array}\right) .
$$

Writing Eq. (7.11) in components we get:

where

$$
\left.\begin{array}{l}
\xi(t, s)=\xi_{\text {in }}\left(t_{0}, t, s\right)+\int_{t_{0}}^{t} G(t+s, \sigma+s) F_{2}(\xi(\sigma, s), \sigma, s) d \sigma \\
\eta(t, s)=\eta_{\text {in }}\left(t_{0}, t, s\right)+\int_{t_{0}}^{t} \frac{\partial G}{\partial t}(t+s, \sigma+s) F_{2}(\xi(\sigma, s), \sigma, s) d \sigma,
\end{array}\right\}
$$

$$
\begin{aligned}
G\left(t, t_{0}\right) & :=\Phi(t) \Psi\left(t_{0}\right)-\Psi(t) \Phi\left(t_{0}\right) \\
& =\Psi(t)\left[W(t)-W\left(t_{0}\right)\right] \Psi\left(t_{0}\right)
\end{aligned}
$$


and

$$
\left.\begin{array}{rl}
\xi_{\text {in }}\left(t_{0}, t, s\right)= & \Psi(t+s)\left(\Phi^{\prime}\left(t_{0}+s\right) \xi\left(t_{0}, s\right)-\Phi\left(t_{0}+s\right) \eta\left(t_{0}, s\right)\right) \\
& +\Phi(t+s)\left(-\Psi^{\prime}\left(t_{0}+s\right) \xi\left(t_{0}, s\right)+\Psi\left(t_{0}+s\right) \eta\left(t_{0}, s\right)\right) \\
= & -\frac{\partial G}{\partial t_{0}}\left(t+s, t_{0}+s\right) \xi\left(t_{0}, s\right)+G\left(t+s, t_{0}+s\right) \eta\left(t_{0}, s\right), \\
\eta_{\text {in }}\left(t_{0}, t, s\right)= & \frac{\partial \xi_{\text {in }}}{\partial t}\left(t_{0}, t, s\right) \\
= & -\frac{\partial^{2} G}{\partial t \partial t_{0}}\left(t+s, t_{0}+s\right) \xi\left(t_{0}, s\right)+\frac{\partial G}{\partial t}\left(t+s, t_{0}+s\right) \eta\left(t_{0}, s\right) .
\end{array}\right\}
$$

We also can write (7.12) in the following way:

$$
\left.\begin{array}{l}
\xi(t, s)=\xi^{1}(t)+\int_{t_{0}}^{t} G(t+s, \sigma+s) g(\sigma+s, \xi(\sigma, s)) d \sigma, \\
\eta(t, s)=\eta^{1}(t)+\int_{t_{0}}^{t} \frac{\partial G}{\partial t}(t+s, \sigma+s) g(\sigma+s, \xi(\sigma, s)) d \sigma,
\end{array}\right\}
$$

with

$$
\left.\begin{array}{l}
\xi^{1}(t)=\xi^{1}\left(t_{0}, t, s\right)=\xi_{\text {in }}\left(t_{0}, t, s\right)+\mu \int_{t_{0}}^{t} G(t+s, \sigma+s) \sin \frac{\sigma}{\varepsilon} d \sigma \\
\eta^{1}(t)=\frac{\partial \xi^{1}}{\partial t}\left(t_{0}, t, s\right)=\eta_{\text {in }}\left(t_{0}, t, s\right)+\mu \int_{t_{0}}^{t} \frac{\partial G}{\partial t}(t+s, \sigma+s) \sin \frac{\sigma}{\varepsilon} d \sigma .
\end{array}\right\}
$$

We can integrate by parts the integrals appearing in (7.16) obtaining new expressions for $\left(\xi^{1}(t), \eta^{1}(t)\right)$ :

$$
\left.\begin{array}{rl}
\xi^{1}(t)= & \xi^{1}\left(t_{0}, t, s\right)=-\mu \varepsilon^{2} \sin \frac{t}{\varepsilon} \\
& -\frac{\partial G}{\partial t_{0}}\left(t+s, t_{0}+s\right)\left[\xi\left(t_{0}, s\right)+\mu \varepsilon^{2} \sin \frac{t_{0}}{\varepsilon}\right] \\
& +G\left(t+s, t_{0}+s\right)\left[\eta\left(t_{0}, s\right)+\mu \varepsilon \cos \frac{t_{0}}{\varepsilon}\right] \\
& -\mu \varepsilon^{2} \int_{t_{0}}^{t} \frac{\partial^{2} G}{\partial t_{0}^{2}}(t+s, \sigma+s) \sin \frac{\sigma}{\varepsilon} d \sigma, \\
\eta^{1}(t)= & \eta^{1}\left(t_{0}, t, s\right)=-\mu \varepsilon \cos \frac{t}{\varepsilon} \\
& -\frac{\partial^{2} G}{\partial t \partial t_{0}}\left(t+s, t_{0}+s\right)\left[\xi\left(t_{0}, s\right)+\mu \varepsilon^{2} \sin \frac{t_{0}}{\varepsilon}\right] \\
& +\frac{\partial G}{\partial t}\left(t+s, t_{0}+s\right)\left[\eta\left(t_{0}, s\right)+\mu \varepsilon \cos \frac{t_{0}}{\varepsilon}\right] \\
& -\mu \varepsilon^{2} \int_{t_{0}}^{t} \frac{\partial^{3} G}{\partial t \partial t_{0}^{2}}(t+s, \sigma+s) \sin \frac{\sigma}{\varepsilon} d \sigma .
\end{array}\right\}
$$


At this moment we write several bounds that will be useful in the sequel. First of all, from the expressions (2.7) for $x_{0}(\tau)$ and (7.3) for $g(\tau, \xi)$, we get the following equalities:

$$
\left.\begin{array}{c}
\tan \frac{x_{0}(\tau)}{2}=\sinh \tau, \\
\sin x_{0}(\tau)=\frac{2 \sinh \tau}{\cosh ^{2} \tau}, \\
\cos x_{0}(\tau)=\frac{1-\sinh ^{2} \tau}{\cosh ^{2} \tau},
\end{array}\right\}
$$

that imply, in terms of $\Psi(\tau)$ given by (7.7):

$$
\begin{aligned}
\left|g\left(\tau, \xi^{1}\right)-g\left(\tau, \xi^{2}\right)\right| & \leqq c\left|\Psi^{2}(\tau)\right|\left(\left|\xi^{1}\right|+\left|\xi^{2}\right|\right)\left|\xi^{1}-\xi^{2}\right| \\
|g(\tau, \xi)| & \leqq c\left|\Psi^{2}(\tau)\right||\xi|^{2}
\end{aligned}
$$

for $|\Re \tau| \leqq T,\left|\xi^{1}\right|,\left|\xi^{2}\right| \leqq 1, c$ being a constant depending only on $T$.

The following bounds are a consequence of (7.9):

$$
\begin{aligned}
& |\Psi(\tau)| \leqq \frac{K}{\left|\tau-\frac{\pi}{2} i\right|}, \quad\left|\Psi^{\prime}(\tau)\right| \leqq \frac{K}{\left|\tau-\frac{\pi}{2} i\right|^{2}}, \quad\left|\Psi^{\prime \prime}(\tau)\right| \leqq \frac{K}{\left|\tau-\frac{\pi}{2} i\right|^{3}} \\
& \frac{1}{K}\left|\tau-\frac{\pi}{2} i\right|^{2} \leqq|\Phi(\tau)| \leqq K\left|\tau-\frac{\pi}{2} i\right|^{2}, \\
& \left|\Phi^{\prime}(\tau)\right| \leqq K\left|\tau-\frac{\pi}{2} i\right|, \quad\left|\Phi^{\prime \prime}(\tau)\right| \leqq K
\end{aligned}
$$

for $|\mathfrak{R} \tau| \leqq T, 0 \leqq \mathfrak{I} \tau \leqq \frac{\pi}{2}$, with $K$ a constant depending only on $T$.

The following lemma will be used in the proof of the next two propositions:

Lemma 7.1. Let $\bar{t}, t \in \mathbb{R}, s \in \mathbb{C}$ such that

$$
-T \leqq \bar{t}+\mathfrak{R} s \leqq t+\mathfrak{R} s \leqq T, \quad 0 \leqq \mathfrak{I} s \leqq \frac{\pi}{2}-\varepsilon
$$

and let us introduce the notation

$$
\rho_{[\bar{t}, t]}^{-2}(s):=\sup \frac{2}{\left|\sigma+s-\frac{\pi}{2} i\right|^{2}}
$$

where the supremum is taken for $\sigma \in[\bar{t}, t]$. Then the following inequalities hold:
a) $\int_{\bar{t}}^{t}\left|\frac{\partial^{2} G}{\partial t_{0}^{2}}(t+s, \sigma+s) \sin \frac{\sigma}{\varepsilon}\right| d \sigma \leqq P\left(|\Psi(t+s)|+\rho_{[\bar{t}, t]}^{-2}(s)|\Phi(t+s)|\right)$,
b) $\int_{\bar{t}}^{t}\left|G(t+s, \sigma+s) \Psi^{2}(\sigma+s)\right| d \sigma \leqq P\left(|\Psi(t+s)|+\rho_{[\bar{t}, t]}^{-2}(s)|\Phi(t+s)|\right)$, 

c) $\int_{\bar{t}}^{t}\left|\frac{\partial^{3} G}{\partial t \partial t_{0}^{2}}(t+s, \sigma+s) \sin \frac{\sigma}{\varepsilon}\right| d \sigma \leqq P\left(\left|\Psi^{\prime}(t+s)\right|+\rho_{[\bar{t}, t]}^{-2}(s)\left|\Phi^{\prime}(t+s)\right|\right)$,
d) $\int_{\bar{t}}^{t}\left|\frac{\partial G}{\partial t}(t+s, \sigma+s) \Psi^{2}(\sigma+s)\right| d \sigma \leqq P\left(\left|\Psi^{\prime}(t+s)\right|+\rho_{[\bar{t}, t]}^{-2}(s)\left|\Phi^{\prime}(t+s)\right|\right)$,

$P$ being a constant depending only on $T$.

Proof. It follows easily from the form of $G$ and the bounds on $\Phi(t+s), \Psi(t+s)$, if we prove the following inequality

$$
\int_{\bar{t}}^{t} \frac{1}{\left|\sigma+s-\frac{\pi}{2} i\right|^{3}} d \sigma \leqq \rho_{[\bar{t}, t]}^{-2}(s) .
$$

Considering first the case $\bar{t}+\mathfrak{R} s \leqq t+\mathfrak{R} s \leqq 0$,

$$
\begin{aligned}
\int_{\bar{t}}^{t} \frac{1}{\left|\sigma+s-\frac{\pi}{2} i\right|^{3}} d \sigma & \leqq \int_{-\infty}^{t} \frac{1}{\left|\sigma+s-\frac{\pi}{2} i\right|^{3}} d \sigma \\
& =\frac{1}{\left(\frac{\pi}{2}-\mathfrak{I} s\right)^{2}}\left[\frac{t+\mathfrak{R} s}{\sqrt{(t+\mathfrak{R} s)^{2}+\left(\frac{\pi}{2}-\mathfrak{I} s\right)^{2}}}+1\right],
\end{aligned}
$$

and therefore,

$$
\left|t+s-\frac{\pi}{2} i\right|^{2} \int_{\bar{t}}^{t} \frac{1}{\left|\sigma+s-\frac{\pi}{2} i\right|^{3}} d \sigma \leqq\left(x^{2}+1\right)\left[\frac{x}{\sqrt{x^{2}+1}}+1\right]:=f(x),
$$

where $x=\frac{t+\mathfrak{R} s}{\frac{\pi}{2}-\mathfrak{I} s}$. Since $f(x) \leqq 1$ for $x \in(-\infty, 0]$, we get

$$
\int_{\bar{t}}^{t} \frac{1}{\left|\sigma+s-\frac{\pi}{2} i\right|^{3}} d \sigma \leqq \frac{1}{\left|t+s-\frac{\pi}{2} i\right|^{2}} \leqq \rho_{[\bar{t}, t]}^{-2}(s) .
$$

When $\bar{t}+\mathfrak{R} s<0<t+\mathfrak{R} s$ we have

$$
\int_{\bar{t}}^{t} \frac{1}{\left|\sigma+s-\frac{\pi}{2} i\right|^{3}} d \sigma \leqq \frac{2}{\left|\frac{\pi}{2}-\mathfrak{I} s\right|^{2}}=\rho_{[\bar{t}, t]}^{-2}(s) .
$$

The other two cases are symmetric.

We are now going to continue $(\xi(t, s), \eta(t, s))$ for values of $(t, s)$ satisfying $t \in \mathbb{R}$, $s \in \mathbb{C}$, and: 


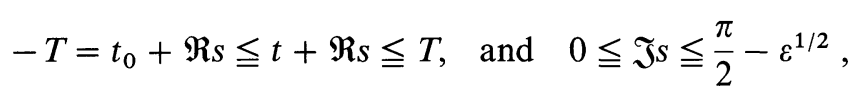

or

$$
-T=t_{0}+\mathfrak{R} s \leqq t+\mathfrak{R} s \leqq t_{1}+\mathfrak{R} s=\varepsilon^{1 / 2}, \text { and } \quad \frac{\pi}{2}-\varepsilon^{1 / 2} \leqq \mathfrak{I} s \leqq \frac{\pi}{2}-\varepsilon .
$$

Proposition 7.2. Let $(\xi(t, s), \eta(t, s))$ be a solution of (7.2) with initial condition on $t_{0}=-T-\mathfrak{R}$ satisfying (7.5). Then, there exists $\mu_{0}>0$, such that for $|\mu|<\mu_{0}$ the solution $(\xi(t, s), \eta(t, s))$ can be extended for $(t, s)$ in the domain (7.23), satisfying the following bounds:

$$
\left.\begin{array}{l}
|\xi(t, s)| \leqq c_{1} \mu \varepsilon \\
|\eta(t, s)| \leqq c_{1} \mu\left(\varepsilon^{2}\left|\Psi^{\prime}(t+s)\right|+\left|\Phi^{\prime}(t+s)\right|\right)
\end{array}\right\}
$$

$c_{1}$ being a constant independent of $\mu$ and $\varepsilon$.

Proof. We shall use the method of successive approximations. We begin the iteration process with $\xi^{0}(t, s)=0$, and consider for $n \geqq 0$ and $(t, s)$ in (7.23) the recurrence suggested by Eq. (7.15):

$$
\xi^{n+1}(t, s)=\xi^{1}(t, s)+\int_{t_{0}}^{t} G(t+s, \sigma+s) g\left(\sigma+s, \xi^{n}(\sigma, s)\right) d \sigma .
$$

In order to carry out this procedure it is necessary to bound accurately $|\Psi(t+s)|$ and $\left|\rho_{\left[t_{0}, t\right]}^{-2}(s) \Phi(t+s)\right|$. Dividing the domain (7.23) in several subdomains, and using (7.22) and Lemma 7.1, we get the following bounds:

a) if $t+\mathfrak{R} \leqq 0$ :

$$
\left.\begin{array}{r}
\rho_{[t, t]}^{-2} \leqq 2\left|t+s-\frac{\pi}{2} i\right|^{-2} \\
|\Psi(t+s)| \leqq K\left|t+s-\frac{\pi}{2} i\right|^{-1} \\
|\Phi(t+s)| \leqq K\left|t+s-\frac{\pi}{2} i\right|^{2},
\end{array}\right\}
$$

b) if $0 \leqq t+\Re s \leqq \varepsilon^{1 / 2}$ and $\frac{\pi}{2}-\varepsilon^{1 / 2} \leqq \mathfrak{I} s \leqq \frac{\pi}{2}-\varepsilon$ :

$$
\left.\begin{array}{rl}
\rho_{\left[t_{0}, t\right]}^{-2} & \leqq 2 \varepsilon^{-2} \\
|\Psi(t+s)| & \leqq K \varepsilon^{-1} \\
|\Phi(t+s)| & \leqq K \varepsilon,
\end{array}\right\}
$$

c) if $0 \leqq t+\mathfrak{R} s \leqq T$ and $0 \leqq \Im s \leqq \frac{\pi}{2}-\varepsilon^{1 / 2}$ :

$$
\left.\begin{array}{rl}
\rho_{\left[t_{0}, t\right]}^{-2} & \leqq 2 \varepsilon^{-1} \\
|\Psi(t+s)| & \leqq K \varepsilon^{-1 / 2} \\
|\Phi(t+s)| & \leqq K
\end{array}\right\}
$$


and consequently, in the whole domain (7.23) we have

$$
\left.\begin{array}{rl}
\rho_{\left[t_{0}, t\right]}^{-2} \cdot \mid & |\Phi(t+s)| \leqq 2 K \varepsilon^{-1} \\
|\Psi(t+s)| \leqq K \varepsilon^{-1} .
\end{array}\right\}
$$

The first iterate in (7.25) is $\xi^{1}(t, s)=\xi^{1}\left(t_{0}, t, s\right)$, as given by (7.17). By the hypothesis (7.5) of Theorem 6 about the initial conditions $\left(\xi\left(t_{0}, s\right), \eta\left(t_{0}, s\right)\right)$, the bounds (7.22) for $G, \partial G / \partial t_{0}$, and Lemma 7.1 with $\bar{t}=t_{0}$, we can bound $\xi^{1}(t, s)$ by:

$$
\begin{aligned}
\left|\xi^{1}(t, s)\right| \leqq & \mu \varepsilon^{2}+2 c_{0} K\left(T^{2}+\frac{\pi^{2}}{4}\right) \mu \varepsilon^{2}|\Psi(t+s)|+2 c_{0} K \frac{1}{T} \mu \varepsilon^{2}|\Phi(t+s)| \\
& +P \mu \varepsilon^{2}|\Psi(t+s)|+P \mu \varepsilon^{2} \rho_{\left[t_{0}, t\right]}^{-2}|\Phi(t+s)|
\end{aligned}
$$

i.e., we get:

$$
\left\|\xi^{1}\right\|:=\sup \left|\xi^{1}(t, s)\right| \leqq \frac{c_{1}}{2} \mu \varepsilon,
$$

where the sup is taken on (7.23). If $n \geqq 1$, using the bound (7.21) for $g$, the Lemma 7.1, and (7.26), we arrive at

$$
\begin{aligned}
\left|\xi^{n+1}(t, s)-\xi^{n}(t, s)\right| & =\left|\int_{t_{0}}^{t} G(t+s, \sigma+s)\left(g\left(\sigma+s, \xi^{n}(\sigma, s)\right)-g\left(\sigma+s, \xi^{n-1}(\sigma, s)\right)\right) d \sigma\right| \\
& \leqq c\left(\left\|\xi^{n}\right\|+\left\|\xi^{n-1}\right\|\right)\left\|\xi^{n}-\xi^{n-1}\right\| \int_{t_{0}}^{t}\left|G(t+s, \sigma+s) \Psi^{2}(\sigma+s)\right| d \sigma \\
& \leqq \frac{3 c K P}{\varepsilon}\left(\left\|\xi^{n}\right\|+\left\|\xi^{n-1}\right\|\right)\left\|\xi^{n}-\xi^{n-1}\right\|
\end{aligned}
$$

If we choose now $\mu_{0}$ small enough $\left(\mu_{0} \leqq \frac{1}{6 c c_{1} K P}\right)$, it follows by induction that for $n \geqq 1,|\mu| \leqq \mu_{0}$,

$$
\begin{aligned}
\left\|\xi^{n}\right\| & \leqq 2\left\|\xi^{1}\right\| \leqq c_{1} \mu \varepsilon \\
\left\|\xi^{n+1}-\xi^{n}\right\| & \leqq \frac{1}{2}\left\|\xi^{n}-\xi^{n-1}\right\|,
\end{aligned}
$$

and consequently $\left(\xi^{n}\right)_{n \geqq 0}$ converges uniformly on (7.23) to the solution $\xi(t, s)$ of (7.15), satisfying the required bound (7.24).

Enlarging $c_{1}$ if necessary, the bound for $\eta(t, s)$ follows from its expression (7.15) and (7.18), in terms of $\xi(t, s)$, and Lemma 7.1.

From the bounds (7.24) we get the following global estimates:

$$
|\xi(t, s)| \leqq c_{1} \mu \varepsilon, \quad|\eta(t, s)| \leqq 2 c_{1} K \mu,
$$

on the domain (7.23). When $\frac{\pi}{2}-\varepsilon^{1 / 2} \leqq \mathfrak{I} s \leqq \frac{\pi}{2}-\varepsilon$, on the final point $t_{1}=\varepsilon^{1 / 2}-\mathfrak{R} s,(7.24)$ gives a better bound for $\eta\left(t_{1}, s\right)$ :

$$
\left|\xi\left(t_{1}, s\right) \leqq c_{1} \mu \varepsilon, \quad\right| \eta\left(t_{1}, s\right) \mid \leqq 2 c_{1} K \mu \varepsilon^{1 / 2} .
$$

Proposition 7.3. Let $s \in \mathbb{C}$ and $t_{1} \in \mathbb{R}$ such that

$$
t_{1}=\varepsilon^{1 / 2}-\mathfrak{R} s, \quad \frac{\pi}{2}-\varepsilon^{1 / 2} \leqq \mathfrak{I} s \leqq \frac{\pi}{2}-\varepsilon .
$$


Let $(\xi(t, s), \dot{\eta}(t, s))$ a solution of (7.2) with initial condition on $t_{1}$ satisfying (7.28). Then, there exists $\mu_{0}>0$, such that for $|\mu|<\mu_{0}$ the solution $(\xi(t, s), \eta(t, s))$ exists also for

$$
t_{1} \leqq t \leqq T-\Re s, \quad \frac{\pi}{2}-\varepsilon^{1 / 2} \leqq \Im s \leqq \frac{\pi}{2}-\varepsilon
$$

and verifies

$$
|\xi(t, s)| \leqq c_{2} \mu, \quad|\eta(t, s)| \leqq c_{2} \mu,
$$

$c_{2}$ being a constant independent on $\mu, \varepsilon$.

Proof. We shall use exactly the same method of successive approximations as in Proposition 7.2, but replacing the initial condition $t_{0}$ by $t_{1}$ :

$$
\xi^{n+1}(t, s)=\xi^{1}\left(t_{1}, t, s\right)+\int_{t_{1}}^{t} G(t+s, \sigma+s) g\left(\sigma+s, \xi^{n}(\sigma, s)\right) d \sigma .
$$

Now, the first iteration is given by $\xi^{1}\left(t_{1}, t, s\right)$ as specified by (7.17), with $t_{1}$ instead of $t_{0}$, and on the strip (7.29) we have

$$
\left.\begin{array}{c}
\rho_{\left[t_{1}, t\right]}^{-2} \leqq 2 \varepsilon^{-1} \\
|\Psi(t+s)| \leqq \frac{K}{\left|t+s-\frac{\pi}{2} i\right|} \leqq \frac{K}{\varepsilon^{1 / 2}} \\
\frac{\varepsilon}{K} \leqq \frac{1}{K}\left|t+s-\frac{\pi}{2} i\right|^{2} \leqq|\Phi(t+s)| \leqq K\left|t+s-\frac{\pi}{2} i\right|^{2}
\end{array}\right\}
$$

Proceeding like in Proposition 7.2, but using now the initial conditions (7.28), we can bound the first iterate on (7.29) as follows:

$$
\begin{aligned}
\left|\xi^{1}(t, s)\right| & \leqq \mu \varepsilon^{2}+3 c_{1} K^{2} \mu|\Phi(t+s)|+3 c_{1} K^{2} \mu \varepsilon^{3 / 2}|\Psi(t+s)| \\
& \leqq 4 c_{1} K^{4} \mu|\Phi(t+s)|:=\frac{c_{2}^{\prime}}{2} \mu|\Phi(t+s)| .
\end{aligned}
$$

In view of this bound, we define the norm

$$
\|\xi\|_{\Phi}:=\sup \frac{|\xi(t, s)|}{|\Phi(t+s)|},
$$

with the supremum taken on the strip (7.29). With this new terminology, (7.32) becomes simply

$$
\left\|\xi^{1}\right\|_{\Phi} \leqq \frac{c_{2}^{\prime}}{2} \mu
$$

For the successives iterates we have:

$$
\frac{\left|\xi^{n+1}(t, s)-\xi^{n}(t, s)\right|}{|\Phi(t+s)|} \leqq c\left(\left\|\xi^{n}\right\|_{\Phi}+\left\|\xi^{n-1}\right\|_{\Phi}\right)\left(\left\|\xi^{n}-\xi^{n-1}\right\|_{\Phi}\right) \cdot I,
$$


where

$$
\begin{aligned}
I & =\frac{1}{|\Phi(t+s)|} \int_{t_{1}}^{t}\left|G(t+s, \sigma+s) \Psi(\sigma+s)^{2} \Phi(\sigma+s)^{2}\right| d \sigma \\
& \leqq \int_{t_{1}}^{t}\left|\Psi(\sigma+s)^{3} \Phi(\sigma+s)^{2}\right| d \sigma+\left|\frac{\Psi(t+s)}{\Phi(t+s)}\right|_{t_{1}}^{t}\left|\Psi(\sigma+s)^{2} \Phi(\sigma+s)^{3}\right| d \sigma \\
& \leqq K^{5} \int_{t_{1}}^{t}\left|\sigma+s-\frac{\pi}{2} i\right| d \sigma+\frac{K^{7}}{\left|t+s-\frac{\pi}{2} i\right|^{3}} \int_{t_{1}}^{t}\left|\sigma+s-\frac{\pi}{2} i\right|^{4} d \sigma \\
& \leqq K^{5} T\left|t+s-\frac{\pi}{2} i\right|+K^{7} T\left|t+s-\frac{\pi}{2} i\right| \leqq 2 K^{7} T^{2}
\end{aligned}
$$

Choosing now $\mu_{0}$ small enough $\left(\mu_{0} \leqq \frac{1}{4 c c_{2}^{\prime} K^{7} T^{2}}\right)$, it follows by induction that for $n \geqq 1$,

$$
\begin{aligned}
\left\|\xi^{n}\right\|_{\Phi} & \leqq 2\left\|\xi^{1}\right\|_{\Phi} \leqq c_{2}^{\prime} \mu \\
\left\|\xi^{n+1}-\xi^{n}\right\|_{\Phi} & \leqq \frac{1}{2}\left\|\xi^{n}-\xi^{n-1}\right\|_{\Phi}
\end{aligned}
$$

and thus $\left(\xi^{n}\right)_{n \geqq 0}$ converges uniformly on the strip (7.29) to the solution $\xi(t, s)$, satisfying the required bound (7.30). Again, the bound for $\eta(t, s)$ follows from its expression (7.15) and (7.18) in terms of $\xi(t, s)$, and Lemma 7.1, replacing $t_{0}$ by $t_{1}$.

Proof of Theorem 6. First consider $0 \leqq \mathfrak{I} s \leqq \frac{\pi}{2}-\varepsilon$. Putting Propositions 7.2 and 7.3 together, as well as the bounds (7.27) produced by Proposition 7.2, we immediately obtain the extension theorem, with the required estimates.

For $-\left(\frac{\pi}{2}-\varepsilon\right) \leqq \mathfrak{J} s \leqq 0$ we only have to choose $a=-\frac{\pi}{2} i$ in the definition of $W(\tau)$, i.e., we choose

$$
\Phi(\tau)=\Psi(\tau) W(\tau)=\frac{1}{2}\left(\left(\tau+\frac{\pi}{2} i\right) \frac{1}{\cosh \tau}+\sinh \tau\right)
$$

in order to get a second solution $\Phi(\tau)$ of the linear system (7.6) with a double zero at $\tau=-\frac{\pi}{2} i$. Bounds (7.22) are now valid for $-\frac{\pi}{2} \leqq \mathfrak{I} s \leqq 0$, and Propositions 7.2, 7.3 follow exactly in the same way, as well as the extension theorem.

Acknowledgements. We would like to thank E. Fontich and C. Simó for helpful discussions and suggestions, and V.I. Lazutkin, V.G. Gelfreich, M.B. Tabanov, A. Benseny and C. Olivé for making their unpublished results available to us. This work has been partially supported by C.I.C.Y.T. Grant P86-527.

\section{References}

[AC89] Amick, C., Ching, S.C.E., Kadanoff, L.P., Rom-Kedar, V.: Beyond all Orders: Singular Perturbation in a Mapping. Preprint, 1989. To appear in J. Nonlin. Anal. 
[AR64]' Arnold, V.I.: Instability of dynamical systems with several degrees of freedom. Doklady Akad Nauk SSSR 156 (1), 581-585 (1964)

[AKN88] Arnold, V.I., Kozlov, V.V., Neishtadt, A.I.: Dynamical systems III. Berlin, Heidelberg, New York: Springer 1988

[BD87] Benseny, A., Delshams, A.: Asymptotic estimates for the splitting of separatrices in systems with slow dynamics. In: European conference on Iteration Theory, Caldes de Malavella. Alsina C. et al. (eds.) Singapore: World Scientific 1989

[BO91] Benseny, A., Olivé, C.: High precision angles between invariant manifolds for rapidly forced hamiltonian systems. Preprint. To appear in proceedings EQUADIFF 91

[Br71] Bruno, A.D.: Analytical form of differential equations. Trans. Moscow Math. Soc. 25, 131-288 (1971)

[Br72] Bruno, A.D.: The analytical form of differential equations II. Trans. Moscow Math. Soc. 26, 199-239 (1972)

[Br89] Bruno, A.D.: Normalization of a hamiltonian system near a cycle or a torus. Russ. Math. Sur. 44 (2), 53-89 (1989)

[DS89] Delshams, A., Seara, T.M.: Medida de la rotura de variedades invariantes en campos del plano. XI. C. E. D. Y. A., Málaga, Spain: 329-333 (1989)

[FS90] Fontich, E., Simó, C.: The splitting of separatrices for analytic diffeomorphisms. Ergod. Th. \& Dyn. Sys. 10, 295-318 (1990)

[Fo91] Fontich, E.: Exponentially small upper bounds for the splitting of separatrices for high frequency periodic perturbation. Preprint, 1991. To appear in J. Non. Anal.: Theory, Methods, and Applications

[Ge90] Gelfreich, V.G.: Splitting of separatrices for the rapidly forced pendulum. Preprint, 1990

[GLT91] Gelfreich, V.G., Lazutkin, V.F., Tabanov, M.B.: Exponentially small splitting in hamiltonian systems. Chaos, 1 (2), 137-142 (1991)

[GH83] Guckenheimer, J., Holmes, P.: Nonlinear oscillations, dynamical systems and bifurcations of vector fields. Appl. Math. Sciences vol. 42, Berlin, Heidelberg, New York: Springer 1983

[HMS88] Holmes, P., Marsden, J., Scheurle, J.: Exponentially small splittings of separatrices with applications to KAM theory and degenerate bifurcations. Contemp. Math. 81, 213-244 (1988)

[La84] Lazutkin, V.F.: Splitting of separatrices for standard Chirikov's mapping. VINITI, N 6372-84, 24 September 1984, (in Russian)

[La91] Lazutkin, V.F.: On the width of the instability zone near the separatrices of a standard mapping. Soviet Math. Dokl. 42 (1), 5-9 (1991)

[LST89] Lazutkin, V.F., Schachmannski, I.G., Tabanov, M.B.: Splitting of separatrices for standard and semistandard mappings. Physica D, 40, 235-248 (1989)

[Me63] Melnikov, V.K.: On the stability of the center for time periodic perturbations. Trans. Moscow Math. Soc. 12, 1-57 (1963)

[Mo56] Moser, J.: The Analytic Invariants of an Area-Preserving Mapping Near a Hyperbolic Fixed Point. Commun. Pure Appl. Math. 9, 673-692 (1956)

[Mo58] Moser, J.: New aspects in the theory of stability of hamiltonian systems. Commun. Pure Appl. Math. 11, 81-114 (1958)

[Ne84] Neishtadt, A.I.: The separation of motions in systems with rapidly rotating phase. J. Appl. Math. Mech. 48 (2), 133-139 (1984)

[Po93] Poincaré, H.: Les méthodes nouvelles de la mécanique celeste (vol. 2). Paris: GauthierVillars 1893

[Sa82] Sanders, J.: Melnikov's method and averaging. Cel. Mech. 28, 171-181 (1982)

[Se91] Seara, T.M.: Estudi de fenòmens homoclínics en sistemes propers a integrables. Ph.D. Thesis, U. Barcelona, 1991

[Si90] Simó, C.: Private communication, 1990

[Zi82] Ziglin, S.L.: Splitting of separatrices, branching of solutions and nonexistence of an integral in the dynamics of a solid body. Trans. Moscow Math. Soc. 41, I: 283-298 (1982) issue 1 
